تأثير إضفة اللماد المضوي للتربة والنبلت علي المفلت النوعية والمحتوى المحني لنبلت الكوسة (Cucurbita pepo L.)

نجيب محمد مسين المغربي'

المؤبة لالمواد الصلبة الذائبة TSS ومحتوى النبلت من NPK

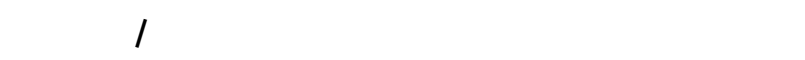

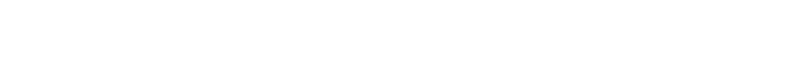

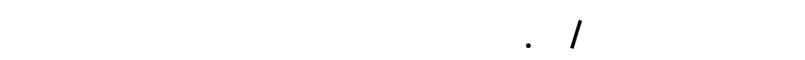

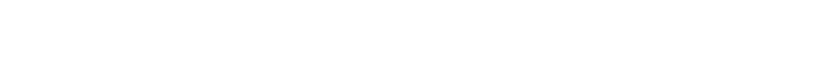
وونن المالة الجلة والمساحة الورقية والنسبة المؤبة لالمواد

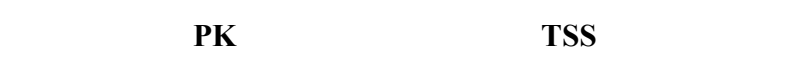

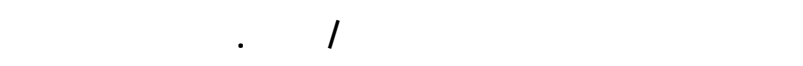

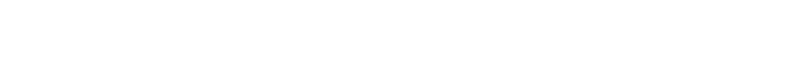
والنسبة المؤبة المواد الصلبة الذائبة TSS عند المعالمالة المعالة كما ألت المعاملة F الجلة والهساحة الورقية وعدد الثمار النبلت ومحتوى النبلت

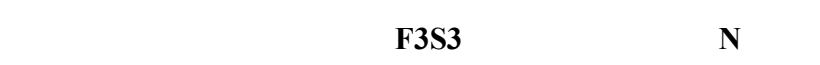
محتوى النبلت من العفور والبوتلسيوم.

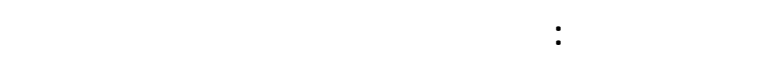
المواد الصلبة الذالبٔبة

$$
\text { الفقم - }
$$

تعتبر الكوسة Squash إحدى أهم محاصيل الخضر التابعة للعائلة القرعية Cucubitaceae وهي من محاصلة الخضر الثائعة الانتشار في الوطن العربي ومنها اليمن.

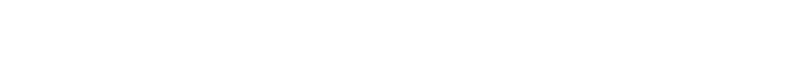
جمبع أنحاء العالم (Dilson, 2002) النتشرت زراعته في اليمن

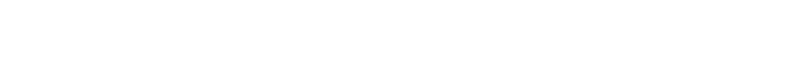
مختلفة وققدر مسلحته في الجمهورية اليمنية وفئه الجائه

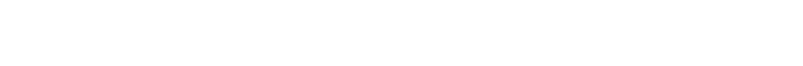

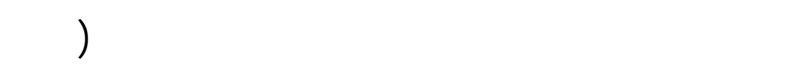

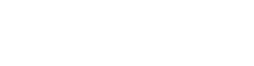

لالرلسة تأثير.طستوبلت مختلفة من السماد المضوي الأرضي واللش عل الهفت النوعية والمحتوى المعني لنبل الكوسة.

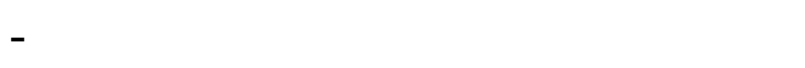

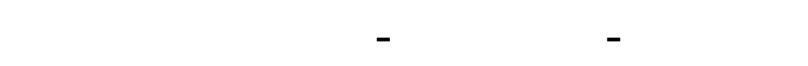

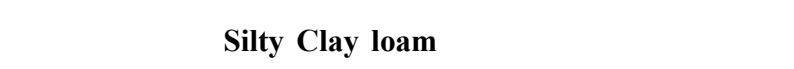

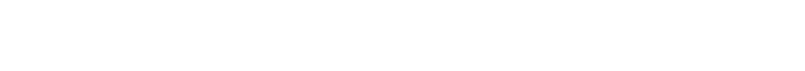

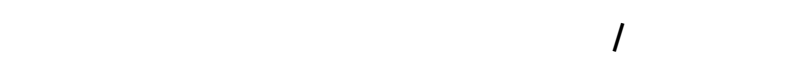

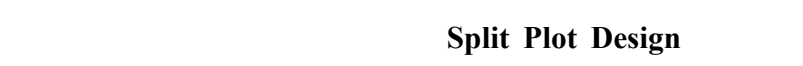

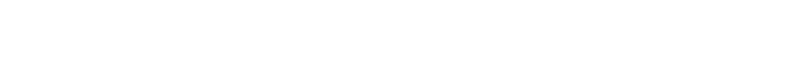
trace elements (Mn, Ca, و 9 gumic substances \%O) B, Mo, Zn, Co, Fe)

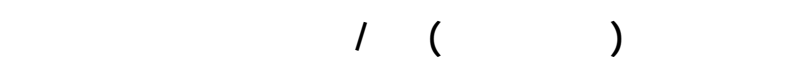
الآتية (F3,F2,F1) عل التوالي ألضف السماد المضوي لأرضي

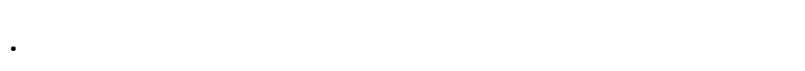

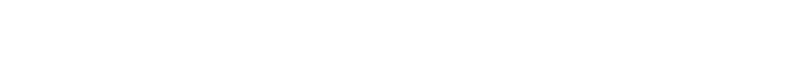

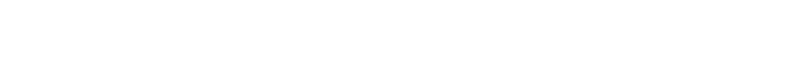

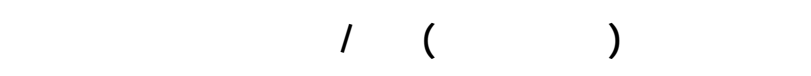

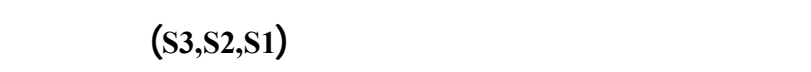

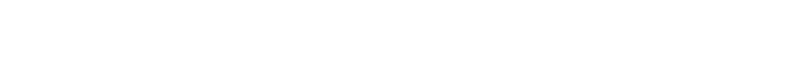

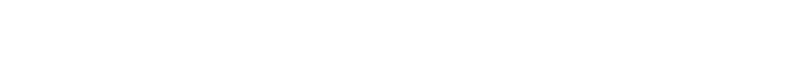
10 يوماً من الرشة الأول جسب مستوبلت الإضلكة المختلفة،

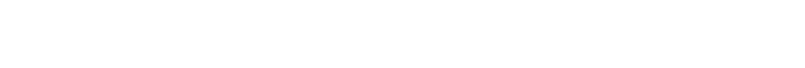

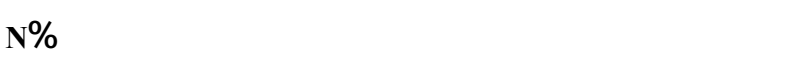

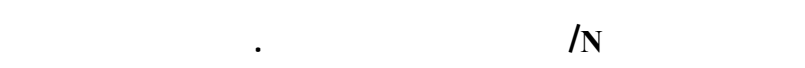

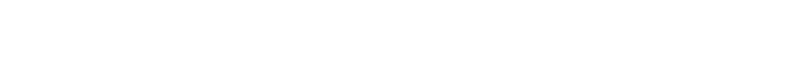
ونن الثمرة وونن الماة الجالة والمساحة الورقية والنسبة المبنة 
أن إضلفة المستخلص البحري SeaForce1 رشا على نبلت

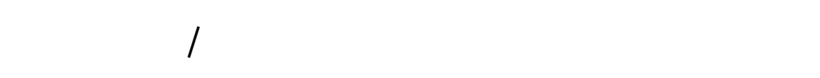
زياة نسبة المواد الصلبة الذائبة في الثمار وكذا زيات الثياة

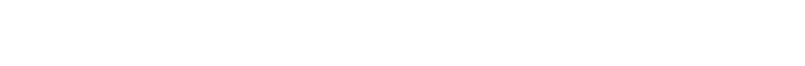

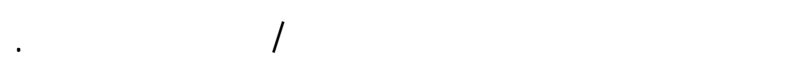

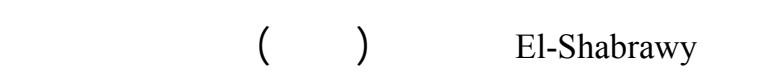

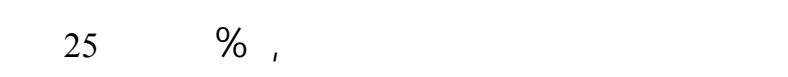

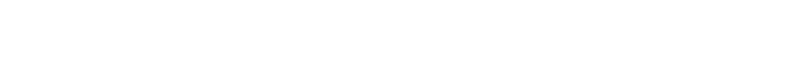

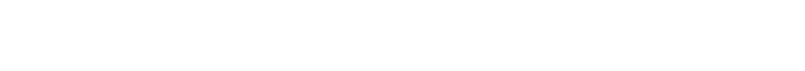

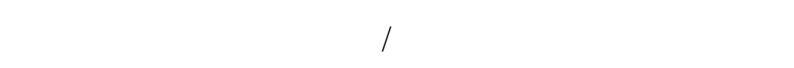

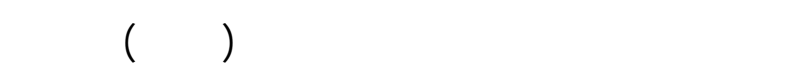

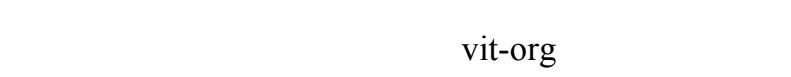
معنوي في زياة المسلحة الورقية وحاطل النبلت كما لاطظ

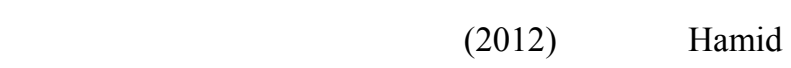

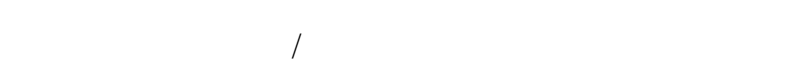

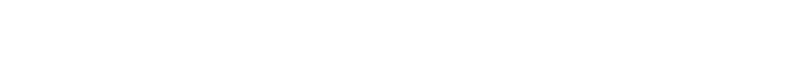

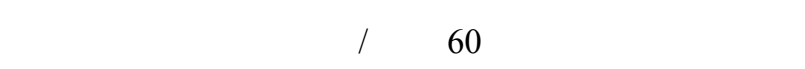
الثمارلنبات ووزن الثمرة والحاطل الكلي للنبت.

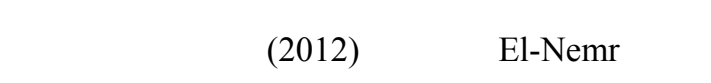

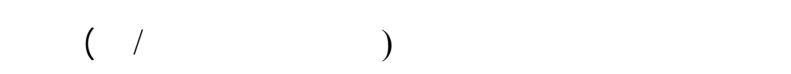

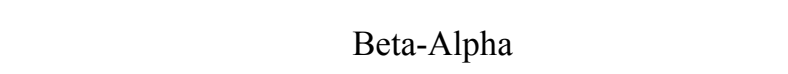

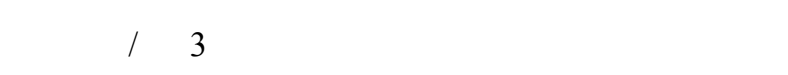

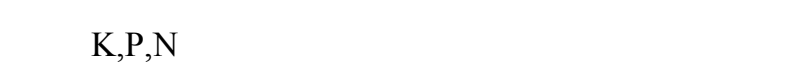

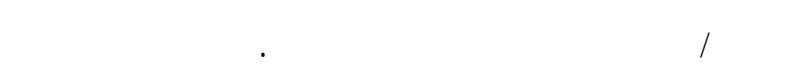

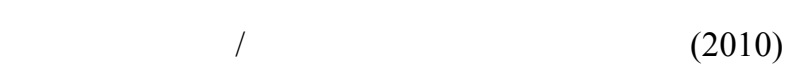

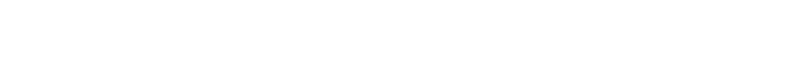

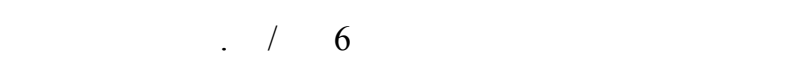

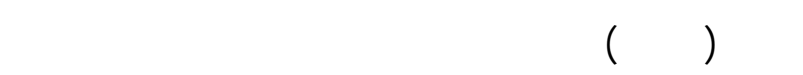

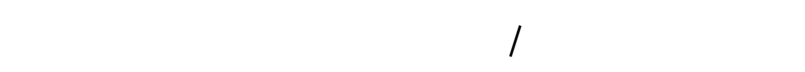

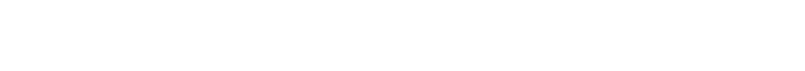

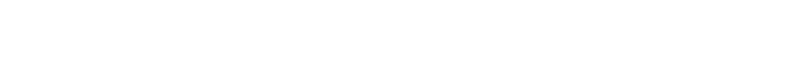

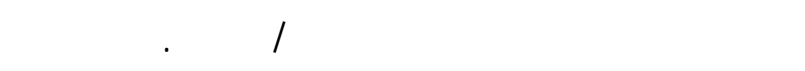

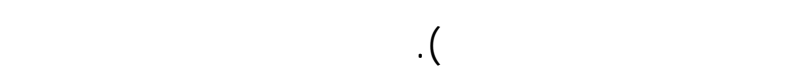
الجالة وشبه الجافة التي تعافي تربها من النخاء النفاض محتواها من المادة العضوية بسب الجفلف وقلة الظطاء النباتي، كما تعاني من الارثفاع النسب لكربونات الكلسيوم وارقفاع إل ـ

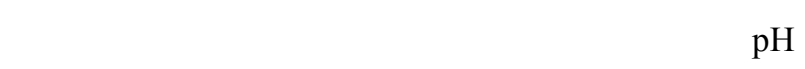

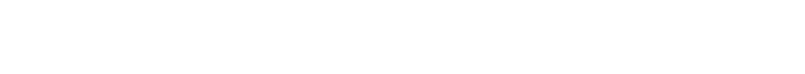

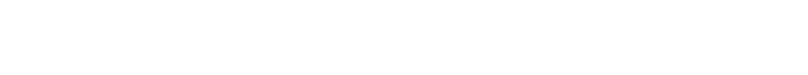

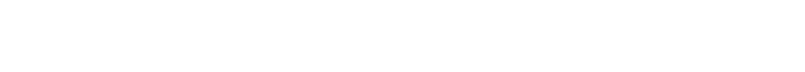

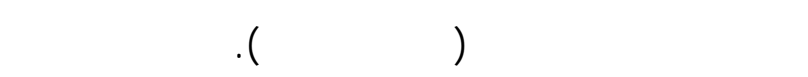

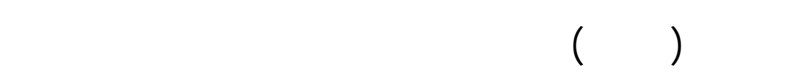

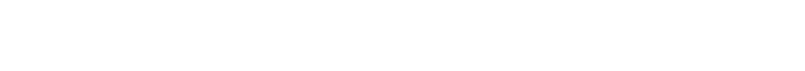

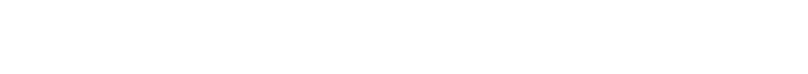

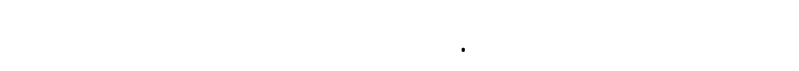

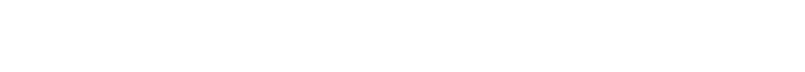

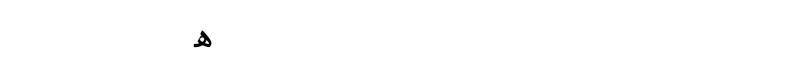

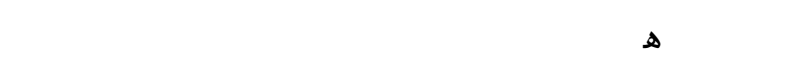

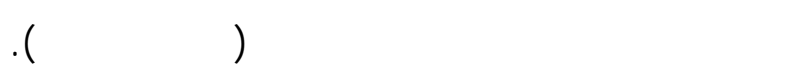

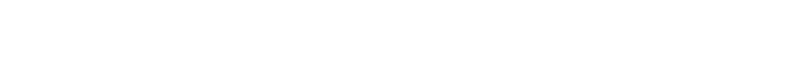

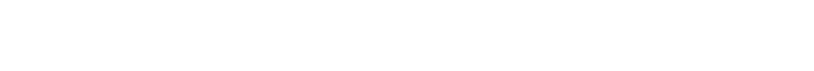

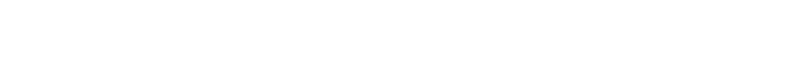

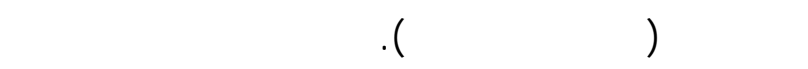
العضويةمل (لحماض الهيوميك والفوليك الدباليه والأحماض لناض

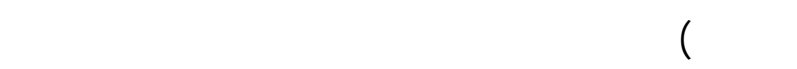

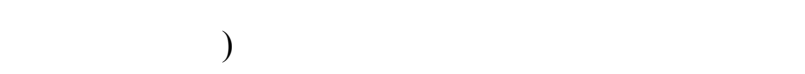
. ( r r o

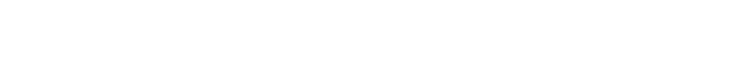

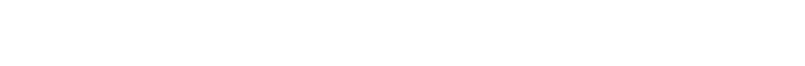

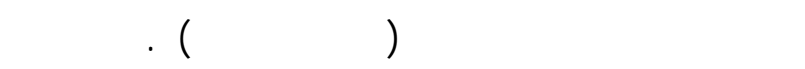
البيلة للأسمدة الكيماوية التوسع في للستخدلم الأسمدة

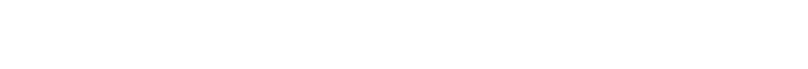
الحفظظ على توفر العناصر الغذائية اللازمة لنمو وإلتناج

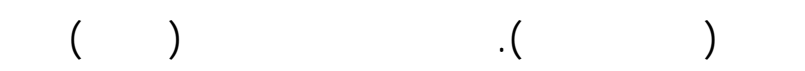


ققليل لمتخدلم الأسمدة الكيماوية ظرا لما تسببه من مضار

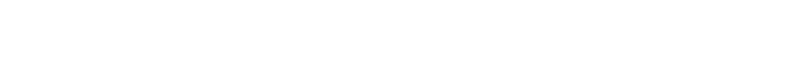
العضوية من المركبلت الطبيعية التي تنميز بعدم سلميتها

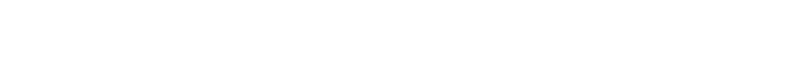

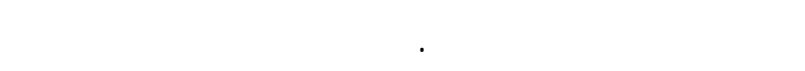

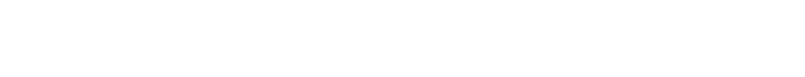

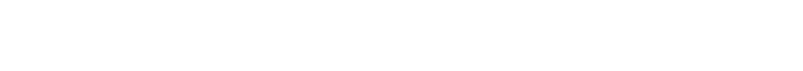
. (Cucurbita pepo L.)

\section{المواد ولرق الملل}

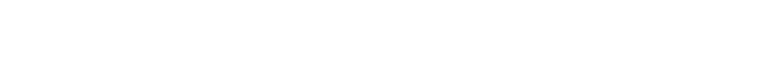

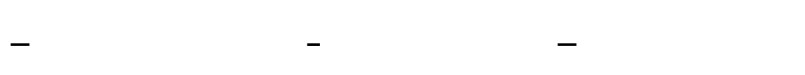

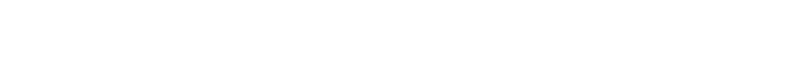
Silty Clay loam

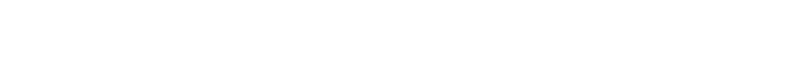
النوعية والمحتوى المعني لنبلت الكوسة.

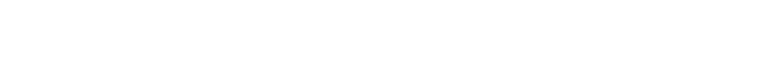

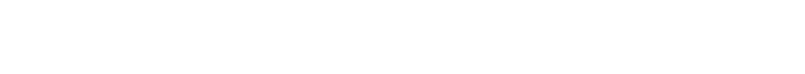

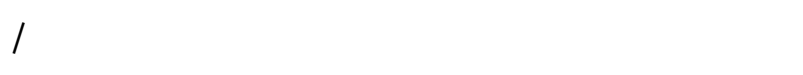

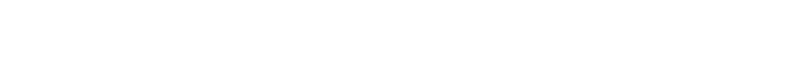

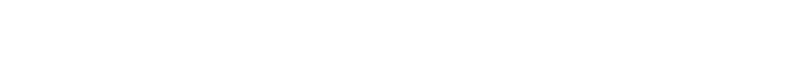

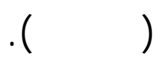

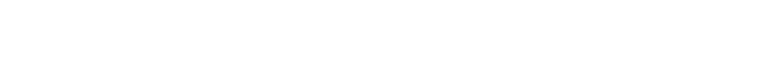
بثلاث مكررات حي عثمث لل الت سميد

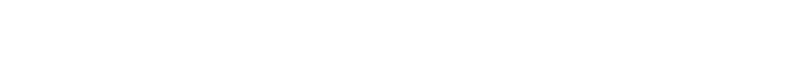
بالرش مل التطع الفرعية وكانت معلملات التجربة كالآتي:
ولخرون(·) Abdel-Mawgoud

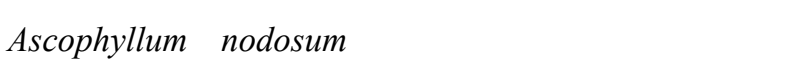

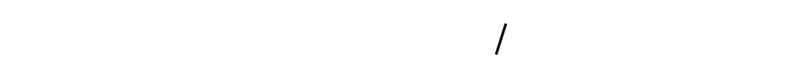

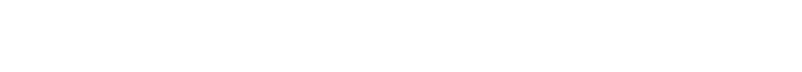
والفنفور والبوتلسيوم في النبت .

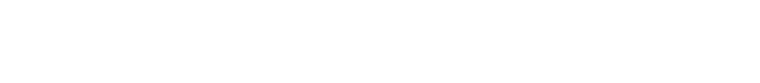

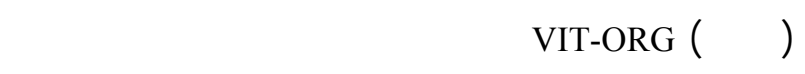

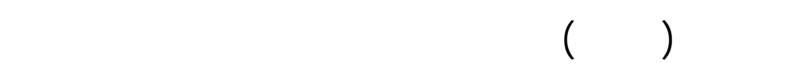

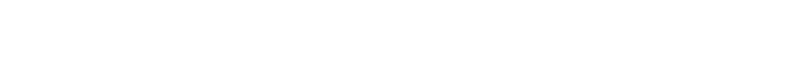

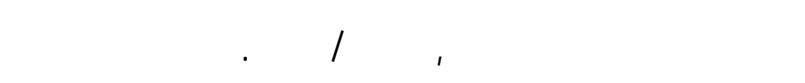

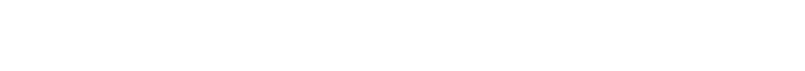

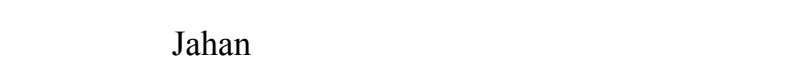

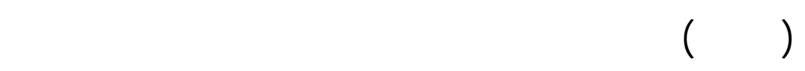

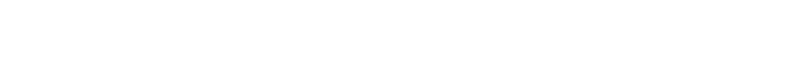

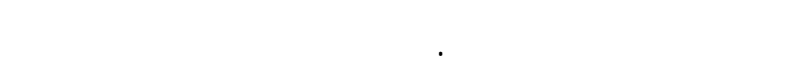

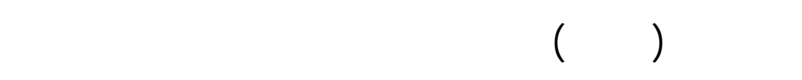

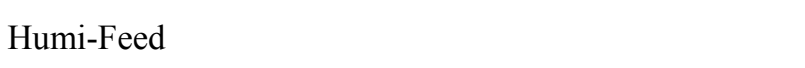

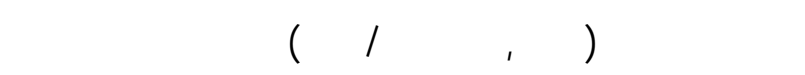

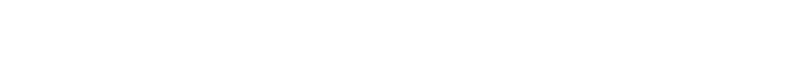

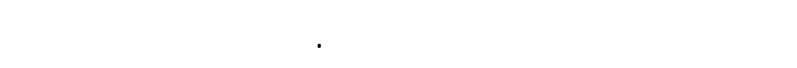

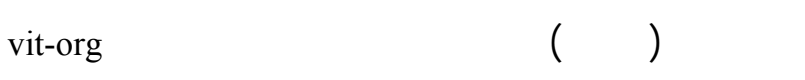

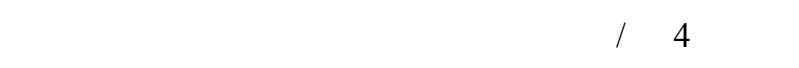

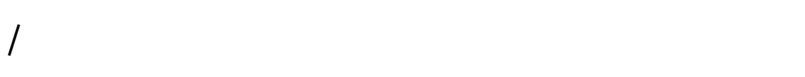

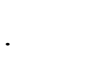

ولأهمية محصول الكوسة والنخفاض إنتلجيته فضلا عن

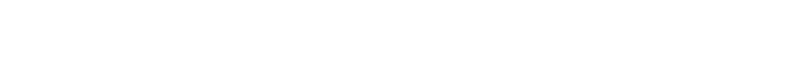
تغنية النبت خصوصا وان التوجه في العالم الآن هو نحو التوري

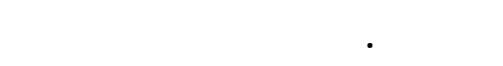

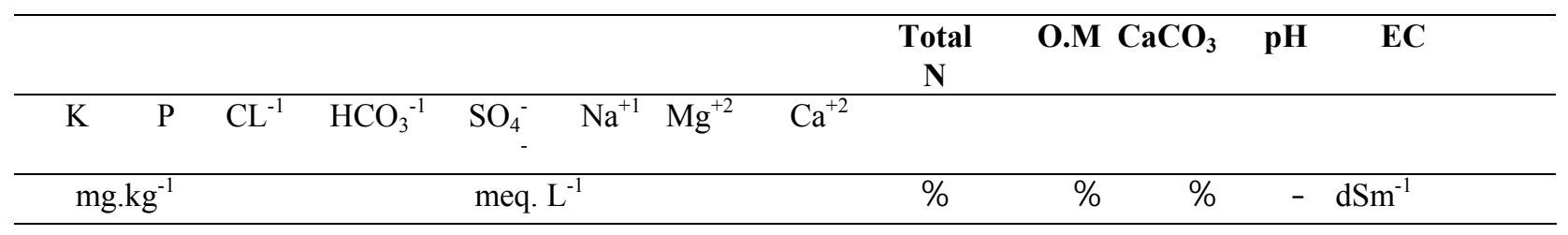




\begin{tabular}{llllllllllllll}
\hline 196 & 7.6 & 1.56 & 1.12 & 1.74 & 1.63 & 0.91 & 1.96 & 0.08 & 0.78 & 7.20 & 7.85 & 0.34 & القيمة
\end{tabular}

بلستعمال جهاز كلداهل، الفنفور الكلي بطريقة مولبي دات

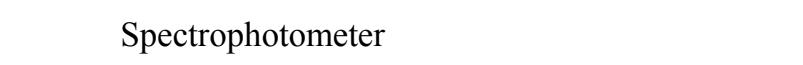
اللطرق الواردة في(Aage et al 1982)، البوتلسيوم الكلي، قدر

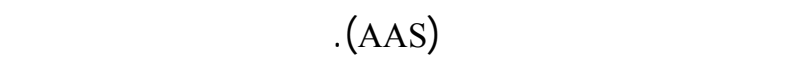

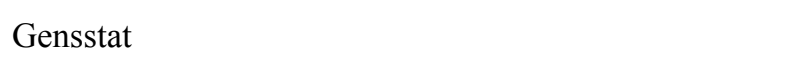
5

\section{النتائج ومنالشتها}

تشير نتنائج التحليل الإحصائي جدول(r) إلى أن إضالفة

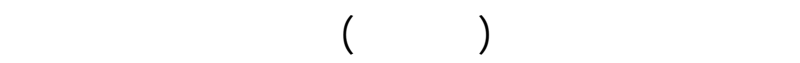

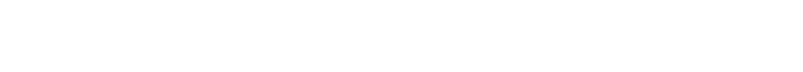
الإضافة عن معلملة المقارنة إذ أعلى المستوى المرنفع من فئل

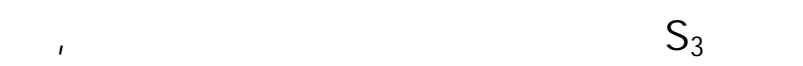

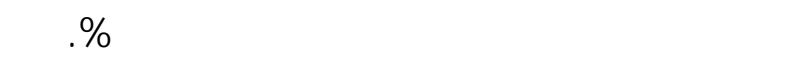

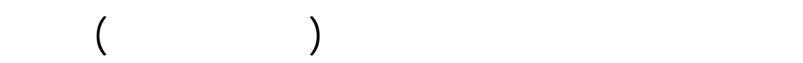

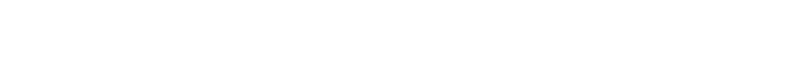

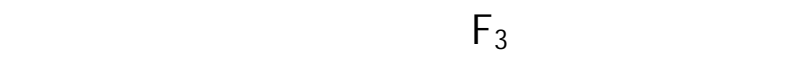

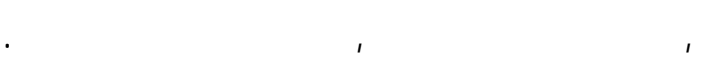

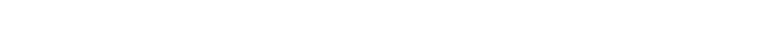
العضوي عنطريق إضلفته للتربة و الرش على النبت أيسا

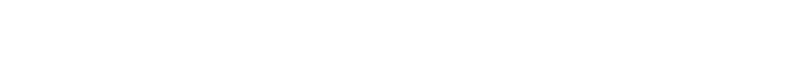
معدلات الإضلفة حيث أعطت المعلملة

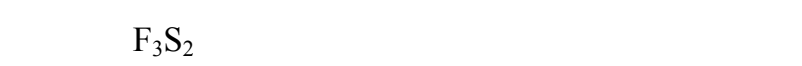

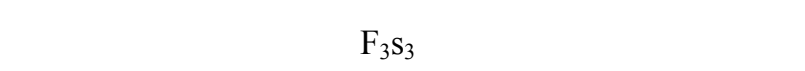

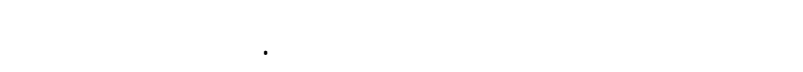

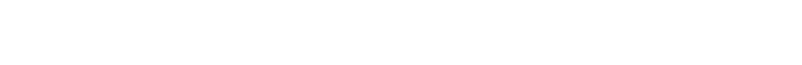
النبلت بالعناصر المغنية الضرورية وخصوصا النت ـروجين والبوتلسيوم والني تساعدفي زياة قوة ونشط النمو الخضري

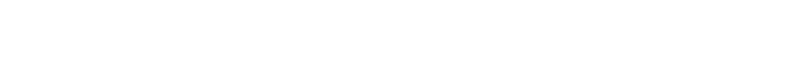

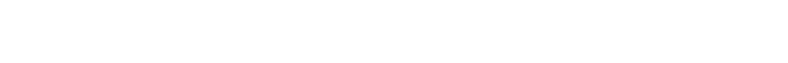

ثلاث م ستويلت م ن ال عسماد الع مضوي ال مصب هـ ي ( ·، ·r، ·ع)طن/هكتار والمسه بيو هو موس والمكون trace elements و و و م (Mn, Ca, B, Mo, Zn, Co, Fe) )، أضيفت للأصص وخططت

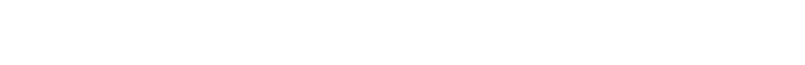
الرموز الآتية (F3, F2, F1) على التوالي وثلاث مـ عستويلت

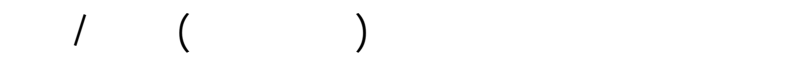

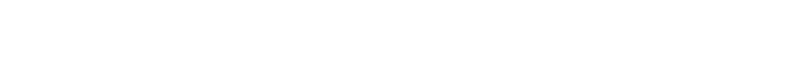

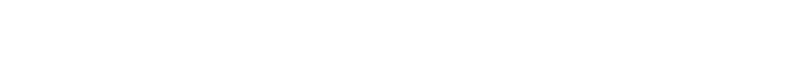

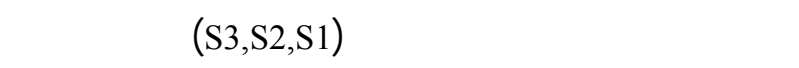

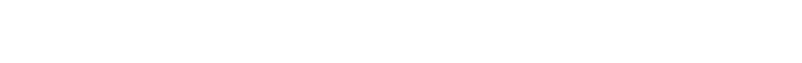

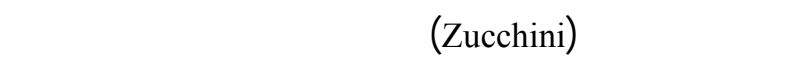

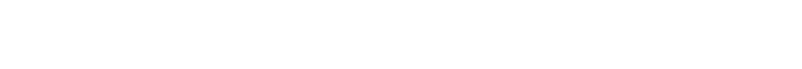

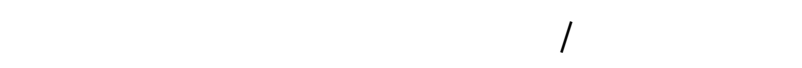

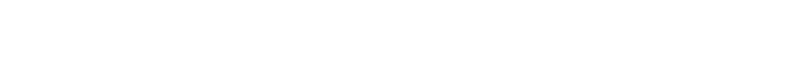

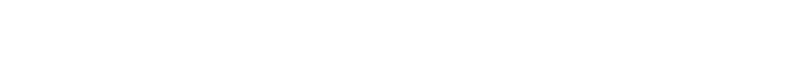

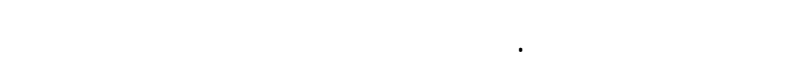

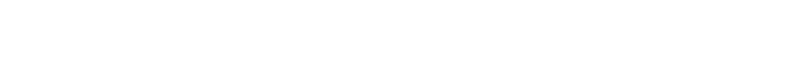

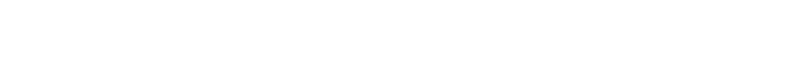

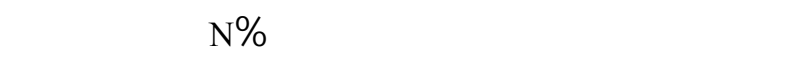

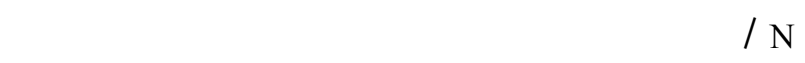

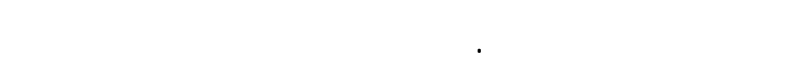

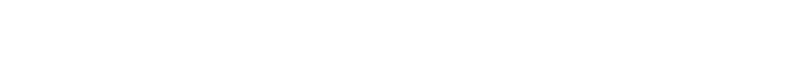

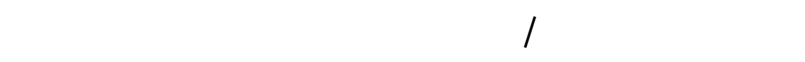

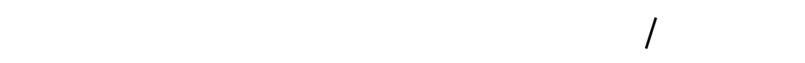

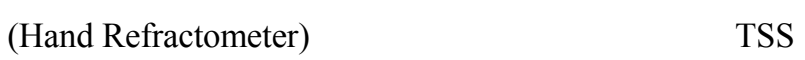
مرطهن النباتلت المجفة في الفرن على درجة 70 درج ه a

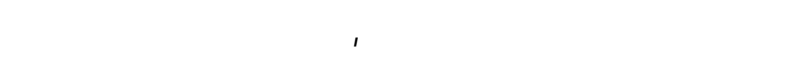

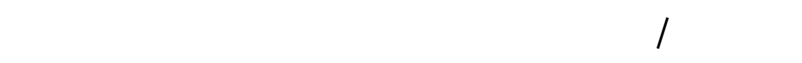
وبيرو مسيد الهيدروجين وقم قق حير النية -روجين الكلا ي 
وبالت الي زي اةة وزن الثم -رة وك ذا حاص لـ النب ـلت

.(ElSahookie,2006)

\begin{tabular}{|c|c|c|c|c|}
\hline MEAN-S & F3 $\left(40\right.$ t. $\left.\mathrm{ha}^{-1}\right)$ & F2 $\left(20\right.$ t. $\left.\mathrm{ha}^{-1}\right)$ & F1 $\left(0\right.$ t.$\left.h a^{-1}\right)$ & $\begin{array}{c}\text { Treatment(mL.L } \\
\text { 1) }\end{array}$ \\
\hline 18.03 & 21.98 & 18.07 & 14.03 & $\mathrm{~S} 1$ \\
\hline 23.49 & 27.97 & 24.00 & 18.50 & $\mathrm{~S} 2$ \\
\hline 27.46 & 26.87 & 25.13 & 30.37 & S3 \\
\hline \multirow[t]{3}{*}{1.423} & & 3.030 & & LSD 0.05 \\
\hline & 25.61 & 22.40 & 20.97 & MEAN- F \\
\hline & & 2.041 & & LSD 0.05 \\
\hline
\end{tabular}

بمتوسط بلغ $\mathrm{F}_{3} \mathrm{~S}_{3} ، \mathrm{~F}_{2} \mathrm{~S}_{2}$

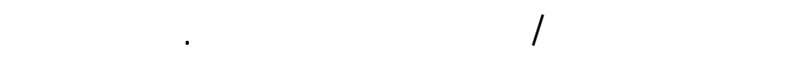

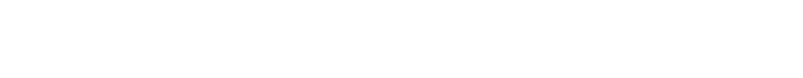

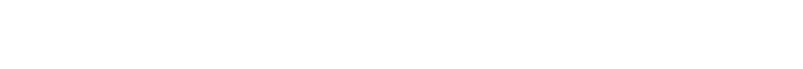

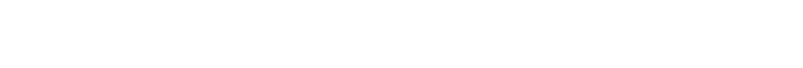

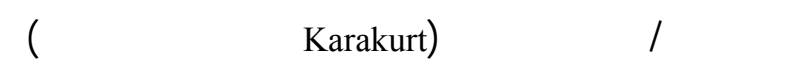

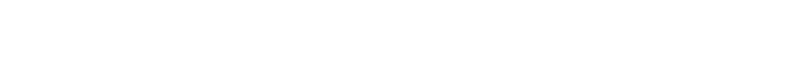

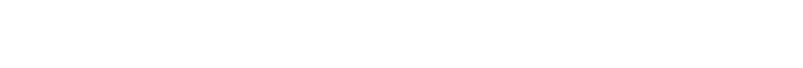

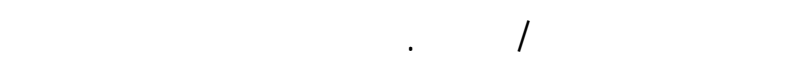

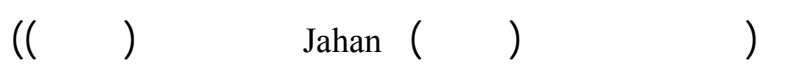
a أن إضافة الأسمدة العضوية بطستويت مختلفة أدت إله حدوث زيادة معنوية في عدد الثمار/ نبلت لنب آلت الكوس إضها

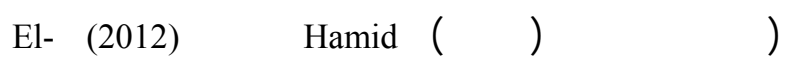

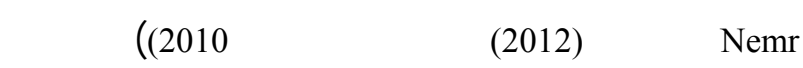

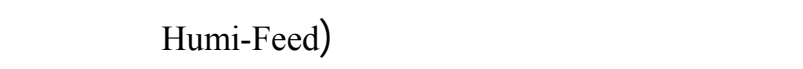
الهيوميك) بترلكيز مختلفة أدت إلى حدوث زيادة معنوية في

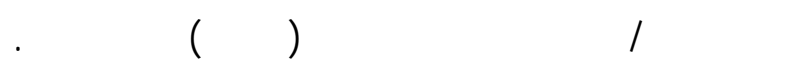
يتضح من الجدول(ع) أن النبة المئوية للمواد ال ـصلبة

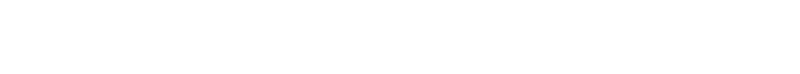

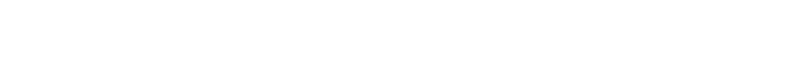
في النبة المئوية للمواد الصلبة الذائبة(TSS) لثمار الكوسة إلمالية

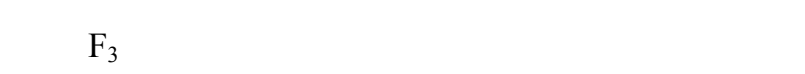

وتقفق النتائج مع ما وجة (التح لفي ولخ _رون (با · r)، Hamid

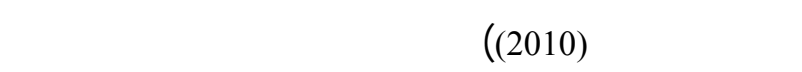

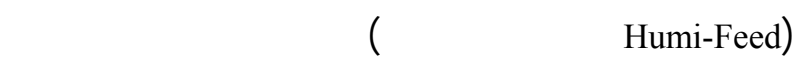

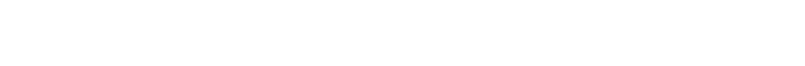

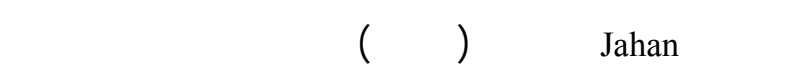

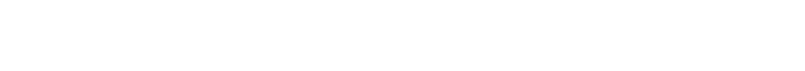
الكوسة. ومجيد( · ( r) على الططا.

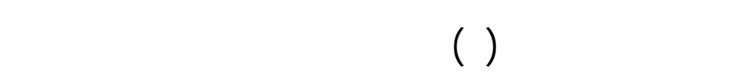

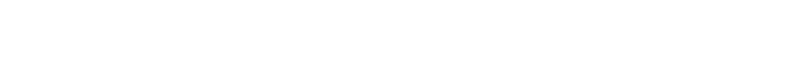

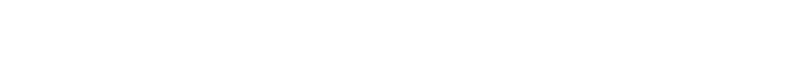

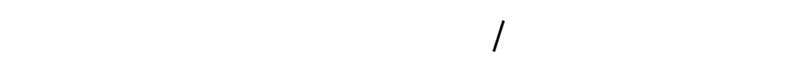

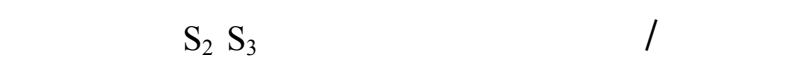
أعطت معلملة المقارنة · ـ ثمار لنبلت.

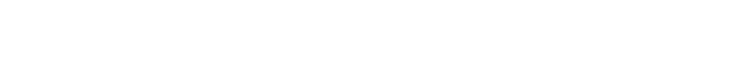

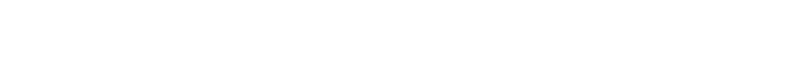

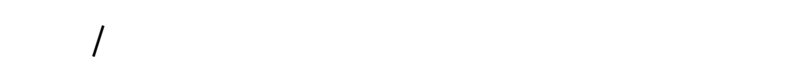

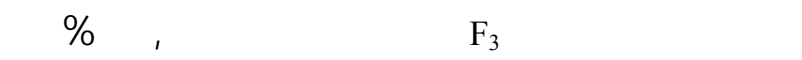

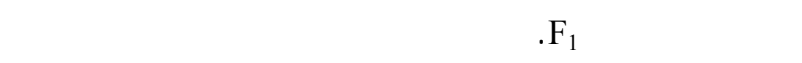

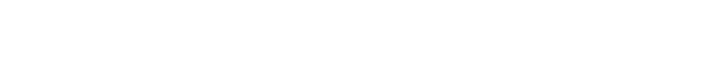
حدوث زيادة معنوية في عدد ثمار الكوسة لنب الت بزيم الة

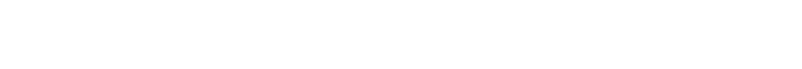
ثمرة لنبك عند المعلملات 


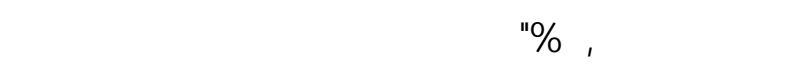
\% \%, rع

جدرب. تأثير إضلفة للسماد المضوي في عدد الثمار/ نبلت

\begin{tabular}{|c|c|c|c|c|}
\hline MEAN S & F3 $\left(40\right.$ t.ha $\left.^{-1}\right)$ & F2 $\left(20\right.$ t.ha $\left.^{-1}\right)$ & F1(0t .ha $\left.{ }^{-1}\right)$ & Treatment(mL.L $\left.{ }^{-1}\right)$ \\
\hline $1 \cdot, \varepsilon \leqslant$ & 13.33 & 9.67 & 8.33 & $\mathrm{~S} 1$ \\
\hline 12.22 & 13.00 & 12.33 & 11.33 & $\mathrm{~S} 2$ \\
\hline IT,YY & 12.33 & 13.00 & 11.33 & S3 \\
\hline \multirow[t]{3}{*}{1.368} & & 1.882 & & LSD 0.05 \\
\hline & 12.89 & $11,7 \mathrm{~V}$ & $1 \cdot, \pi$ & MEAN F \\
\hline & & 1.136 & & LSD 0.05 \\
\hline
\end{tabular}

جلرل ع. تتلثير إلفالة المسماد المضوي في النسبة المئوبة للمواد الصلبة الذائبة TSS في الثمرة

\begin{tabular}{|c|c|c|c|c|}
\hline MEAN. S & F3 $\left(40\right.$ t. $\left.\mathrm{ha}^{-1}\right)$ & F2 $\left(20\right.$ t .ha $\left.{ }^{-1}\right)$ & F1(0 t .hat $\left.{ }^{-1}\right)$ & Treatment $\left(\mathrm{mL} \cdot \mathrm{L}^{-1}\right)$ \\
\hline 2.800 & 3.367 & 2.900 & 2.133 & $\mathrm{~S} 1$ \\
\hline 5.222 & 6.000 & 5.667 & 4.000 & $\mathrm{~S} 2$ \\
\hline 5.400 & 4.733 & 4.867 & 6.600 & S3 \\
\hline \multirow[t]{3}{*}{0.3982} & & 0.5263 & & LSD 0.05 \\
\hline & 4.700 & 4.478 & 4.244 & MEAN. F \\
\hline & & 0.3107 & & LSD 0.05 \\
\hline
\end{tabular}

الذي له دورفي تحفيز والنقل المواد الناتجةمن عملية التمثل

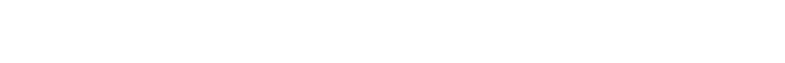

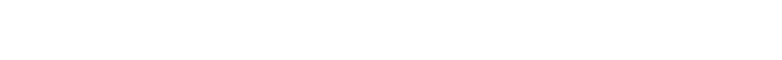

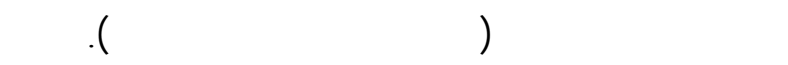

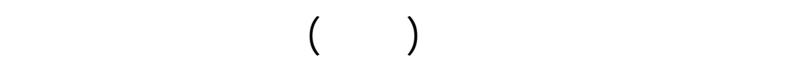

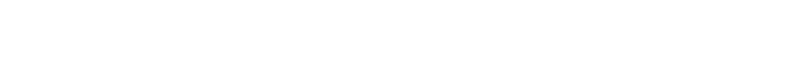

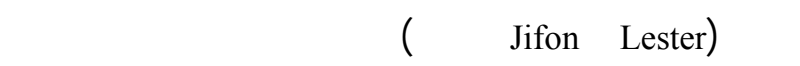
المواد الصلبة الذائبة في ثمارللشملم.

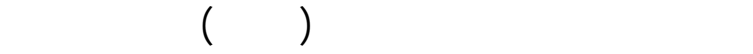
النبلت بالمستخلص البحر، بتركيزمختلفة ادى اله ح دوث زياة معنوية في نسبة المواد الصلبة الذائبة لثمار الكوس ـة ومع كلا من( Abdel-Mawgoud ولخرون( · · (r)، حنشل

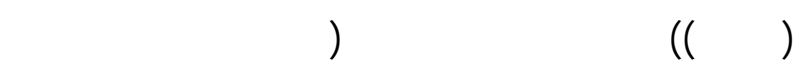

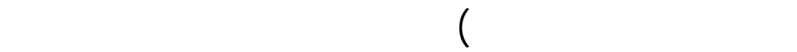

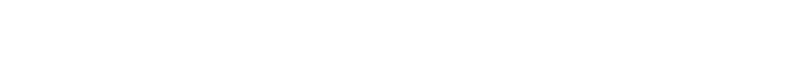

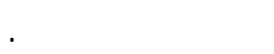

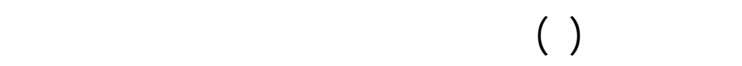

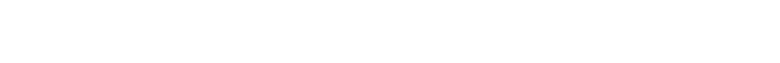

كما أن إضلفة للسماد العضوي عنطريق رش النباتلت

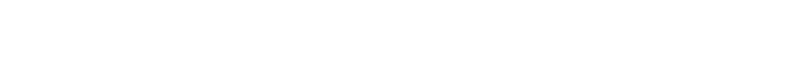
الصلبة الذائبة لثمار الكوسة بـزيادة مستوى الإضافة حي ـثالث

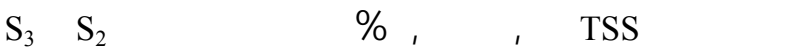

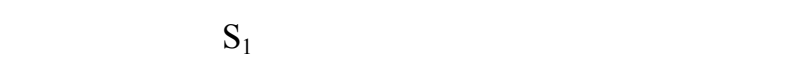

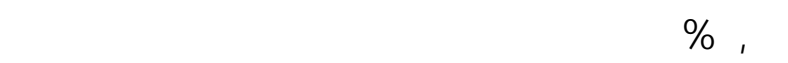

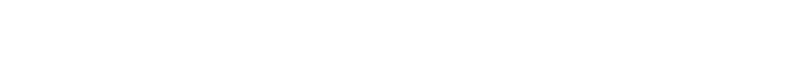

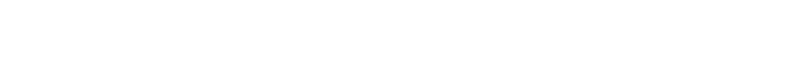

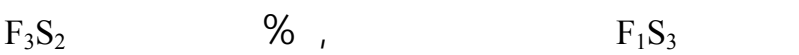
التي أعطت حوالمي 7\% في حن كلتت نسبة TSS في ثمار

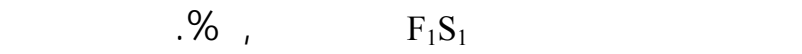

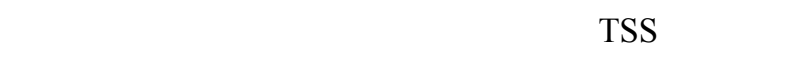
الجيد الذي يؤدي إلى تتجمع المواد الكربوهيدراتيه في الثمار والناتجة من فعالية التمثل الضوئي التي تؤدي إلى زي الة

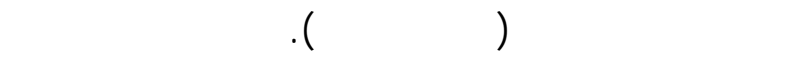

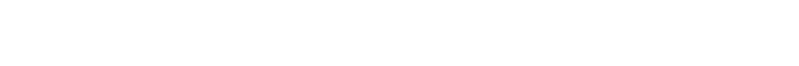

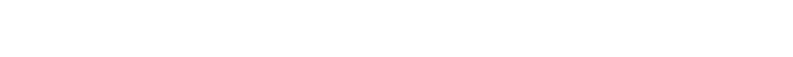

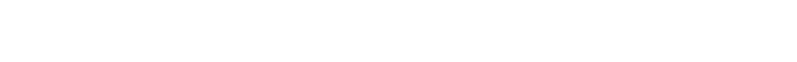

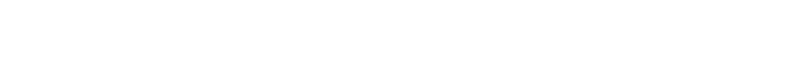


يتضح من الجدول(7) أن المادة الجلفة للنبلت قد تأثرت

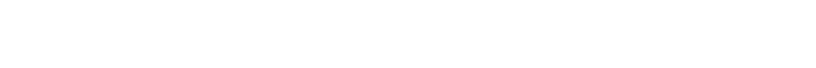

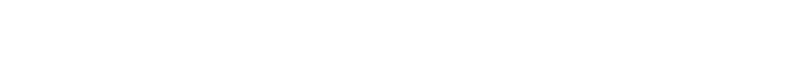

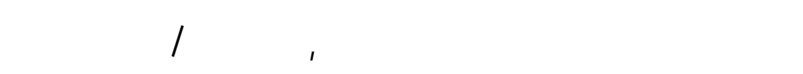

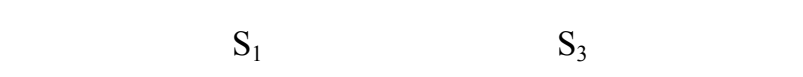

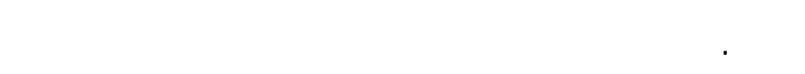

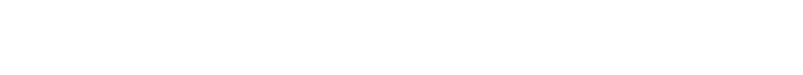

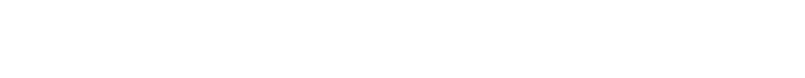

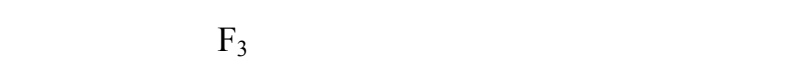

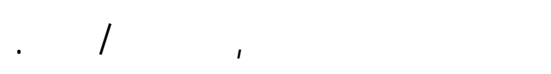

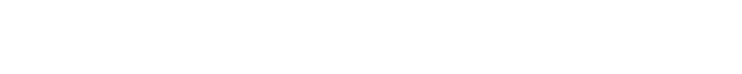

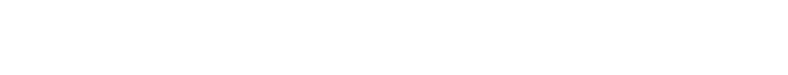

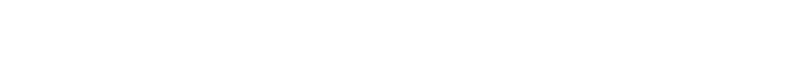
L أعلى $F_{3} S_{3}$

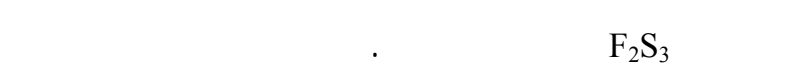
المسلحة الورقية عند الرش بللسماد العضوي إلى الهما تحتويه

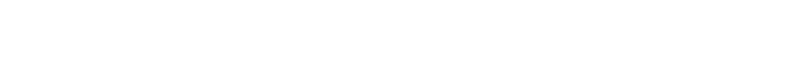

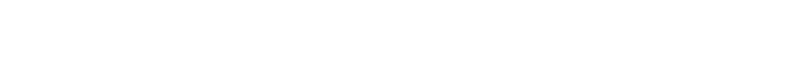

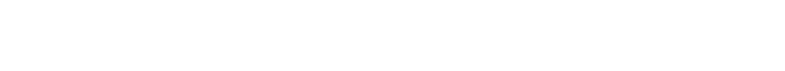

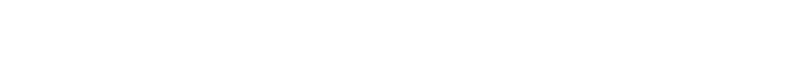

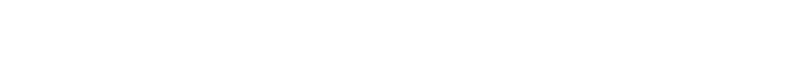

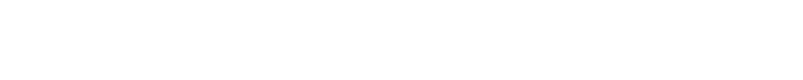

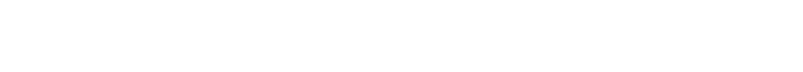

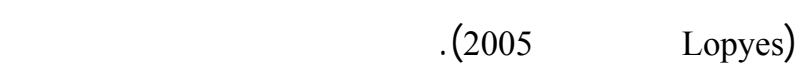

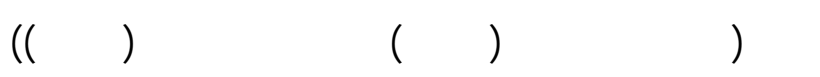

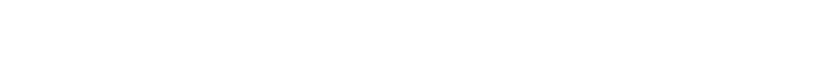

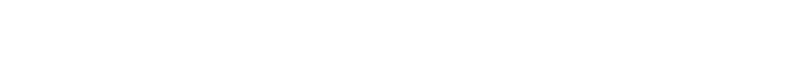

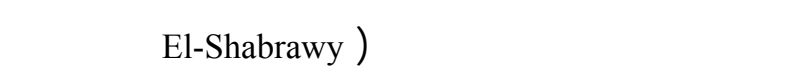

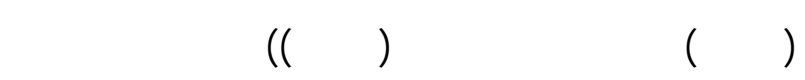

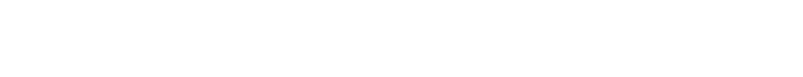

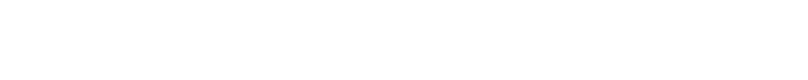
لنبت الخيار.
العضوي عنطريق شش النباتلت إلى حدوث زياة معنوية

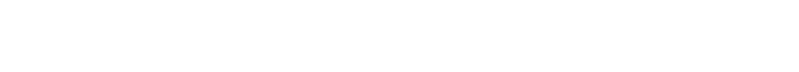

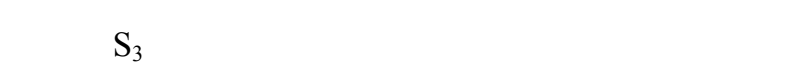

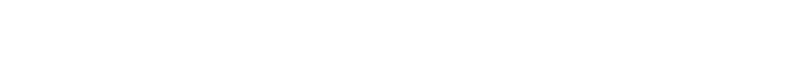

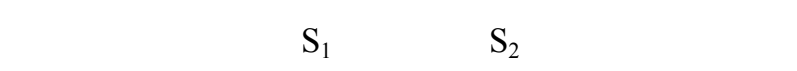

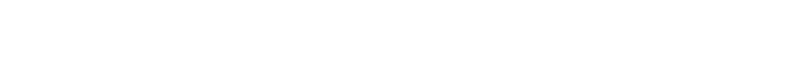

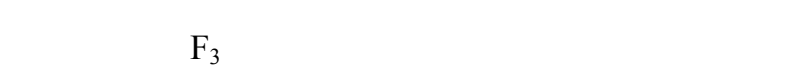

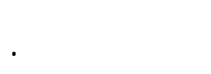

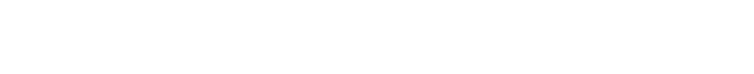

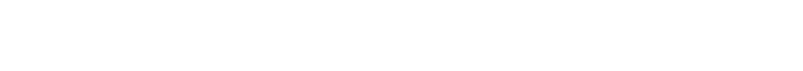

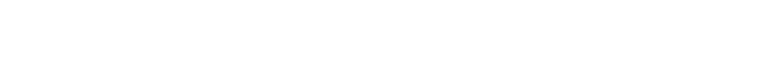
L أعلى $\mathrm{F}_{3} \mathrm{~S}_{3}$

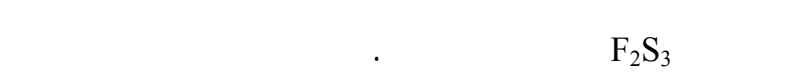

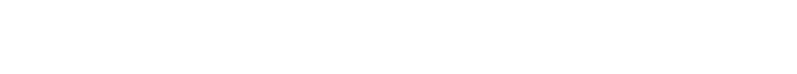

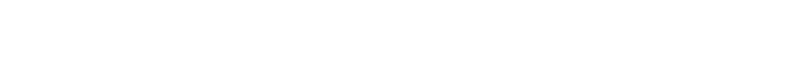

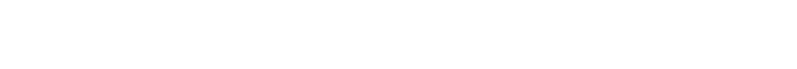

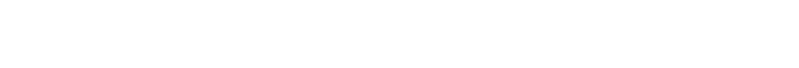

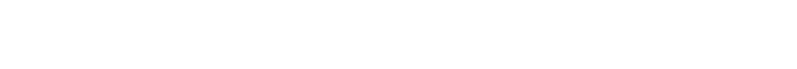
عنمحتواهامن العناصر الغذائية الصغرى والكبرى وهذاميا

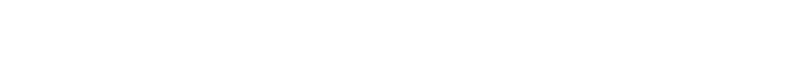

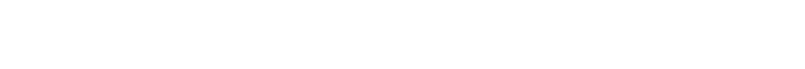

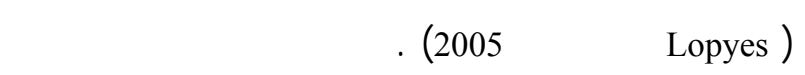

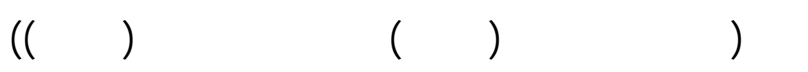

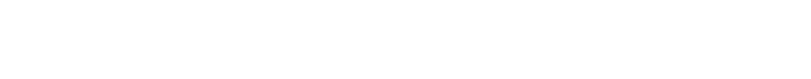

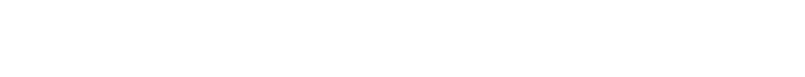

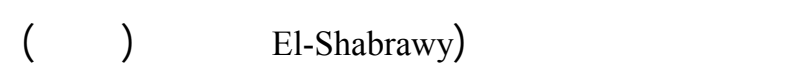

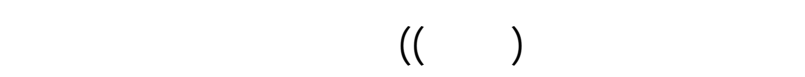

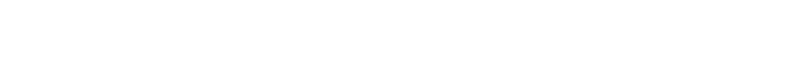

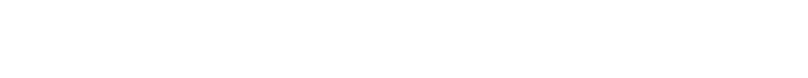

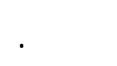


جطط 0. تأثير إضلفة اللماد الهضوي في (متومط المساحة الورقيقسمr

\begin{tabular}{|c|c|c|c|c|}
\hline MEAN. $\mathrm{S}$ & F3 $\left(40\right.$ t. $\left.\mathrm{ha}^{-1}\right)$ & F2 $\left(20 \mathrm{t} \cdot \mathrm{ha}^{-1}\right)$ & F1 $\left(0\right.$ t. $\left.\mathrm{ha}^{-1}\right)$ & Treatment $\left(\mathrm{mL} \cdot \mathrm{L}^{-1}\right)$ \\
\hline 101.24 & 117.17 & 99.07 & 87.50 & $\mathrm{~S} 1$ \\
\hline 164.17 & 186.23 & 167.23 & 139.03 & S2 \\
\hline 215.60 & 224.30 & 220.37 & 202.13 & S3 \\
\hline \multirow[t]{3}{*}{4.176} & & 6.040 & & LSD 0.05 \\
\hline & 175.90 & 162.22 & 142.89 & MEAN. F \\
\hline & & 3.726 & & LSD 0.05 \\
\hline
\end{tabular}

\begin{tabular}{|c|c|c|c|c|}
\hline \multicolumn{5}{|c|}{ 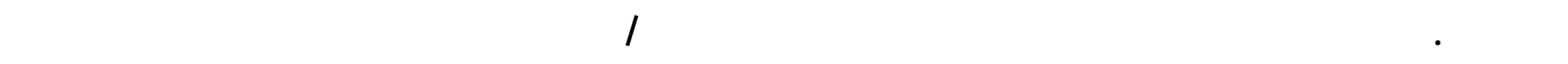 } \\
\hline MEAN .S & F3 (40 t tha-1) & F2 (20 t ha-1) & F1(0 t.ha-1) & Treatment \\
\hline 20.15 & 28.12 & 16.99 & 15.30 & S1 \\
\hline 34.30 & 36.26 & 37.10 & 29.55 & S2 \\
\hline 39.71 & 35.56 & 42.69 & 40.88 & S3 \\
\hline \multirow[t]{3}{*}{3.851} & & 4.082 & & LSD 0.05 \\
\hline & 33.31 & 32.26 & 28.58 & MEAN.F \\
\hline & & 1.867 & & LSD 0.05 \\
\hline
\end{tabular}

النبت والمسلحة الورقية جدول(0) مما أدى إل ع ح دوث زياة معنوية في الوزن الجف للنبلت.

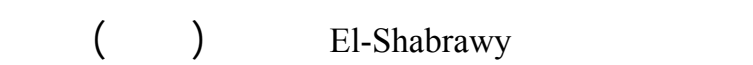

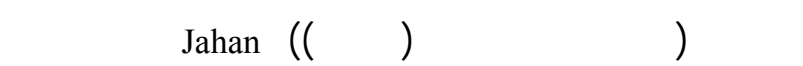

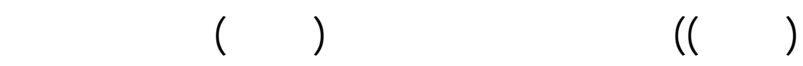

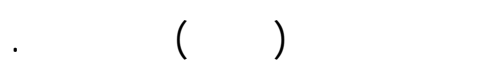
تشير نتائج التحليل الإحصائي جدول(V) إلى أن إضالفة

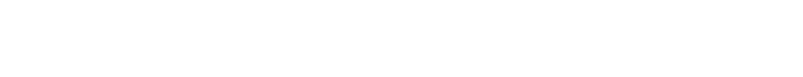

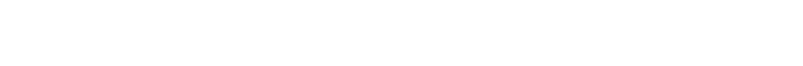

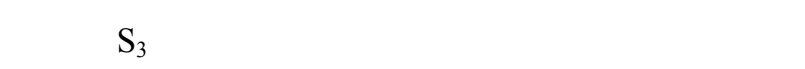

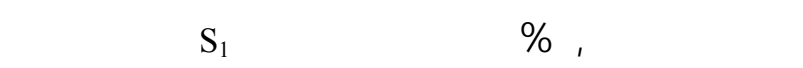

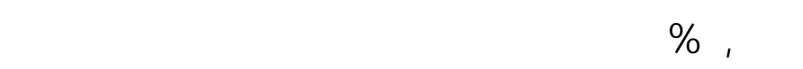
يؤثر معنويا في تركيز النتروجين.

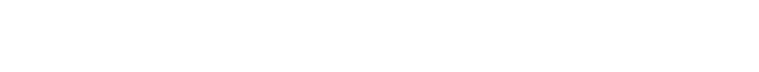

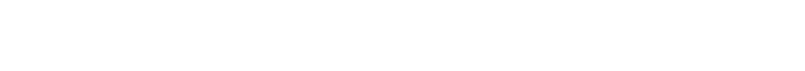
النتروجين للنبلت بزياة مستوى الإضافة وكلن اعلي تركيز

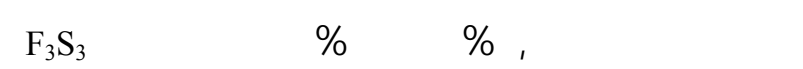

يتضح من الجدول(7) أن المادة الجالة للنبت قد تأثرت

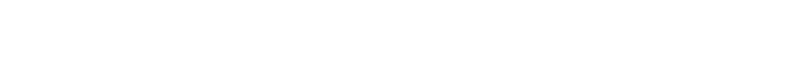

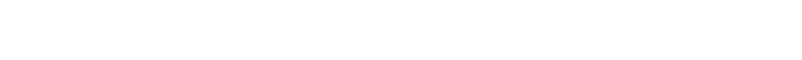

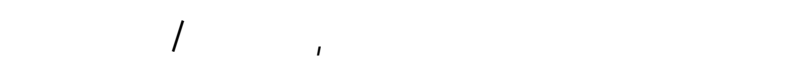

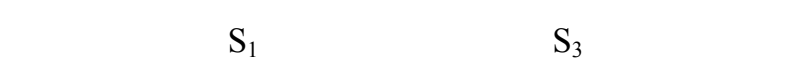

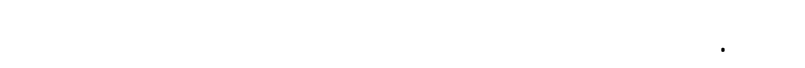

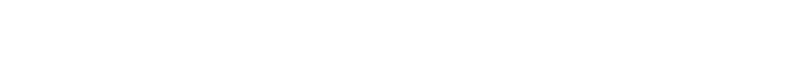

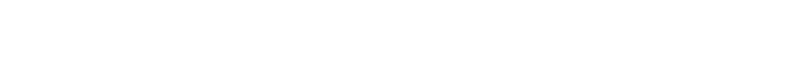

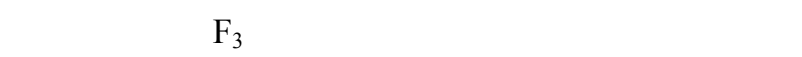

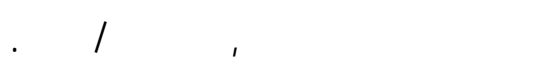

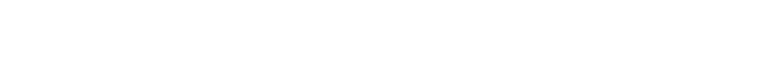

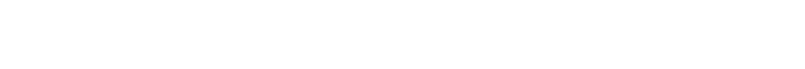

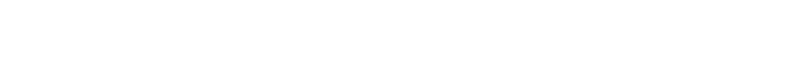

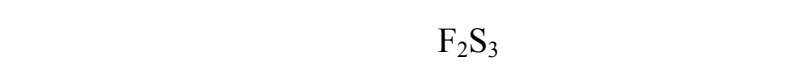

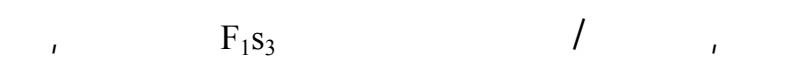

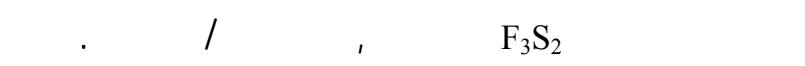

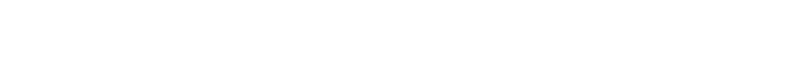

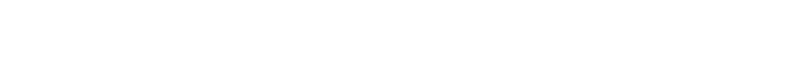




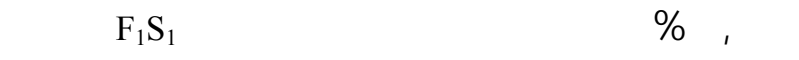
. \% I \%,9

وقد يعزى للسبب في ذلك إلى أن الزكك الموج ـود ف مي الأسمة العضوية المضافة يساهم في بناء البروتين وتنشيط

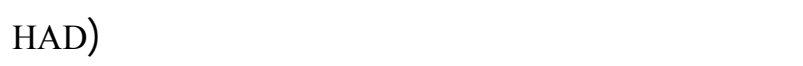
وNADPH) وهذا ما يزيد من ترلكيز النيتروجين والفنفور

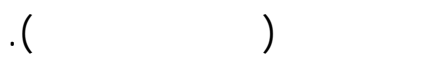
تشير نتائج التحليل الإحصائي جدول(9) إلى أن إضلفة

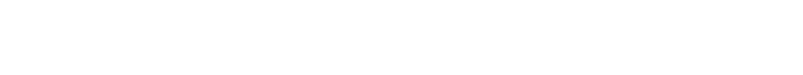

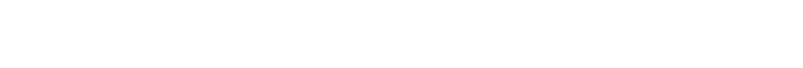

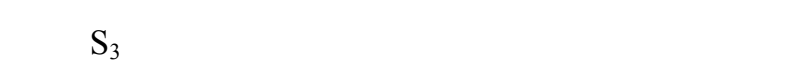

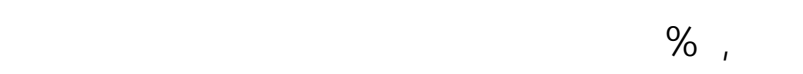

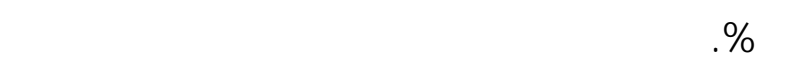

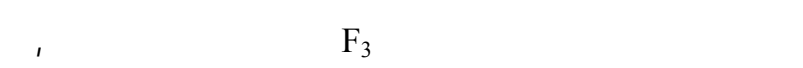

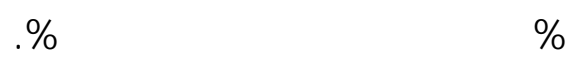

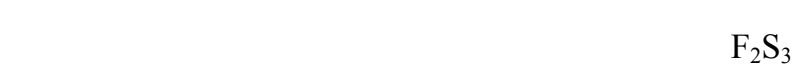
.\% , Vv। تشير نتائج التحليل الإحصائي جدول(^) إلى أن إضلفة

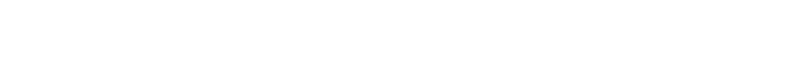

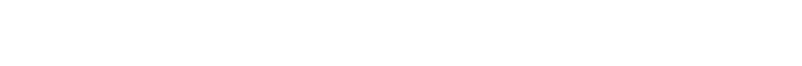

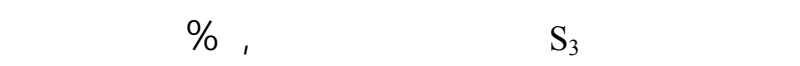

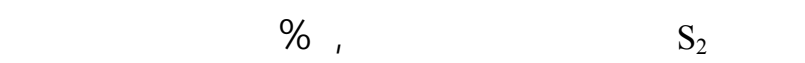

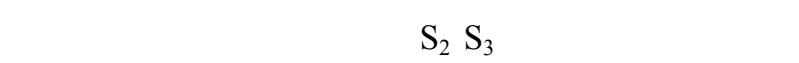
إله التربة أدى إله حدوث زيادة معنوية في تركيز الفنفور

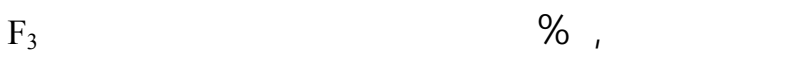

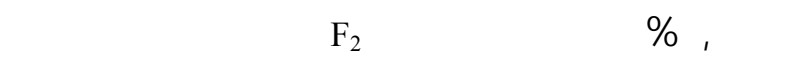

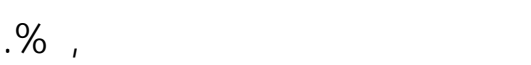

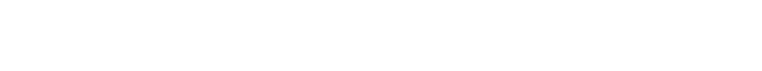

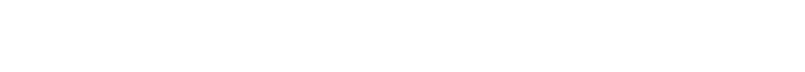

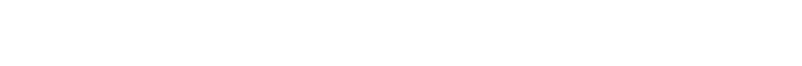
F اعلي تركيز بلغ حسبر, •\% $\mathrm{F}_{3} \mathrm{~S}_{3}$

جدطلv. تأثير إضلفة اللسماد المضوي في تركيز النيتروجن في النبلت

\begin{tabular}{|c|c|c|c|c|}
\hline MEAN S & F3 (40 t .ha-1) & F2 (20 t .ha-1) & F1(0 t .ha-1) & Treatment $\left(\mathrm{mL} \cdot \mathrm{L}^{-1}\right)$ \\
\hline 0.778 & 0.785 & 0.779 & 0.7710 & $\mathrm{~S} 1$ \\
\hline 0.848 & 0.839 & 0.850 & 0.856 & S2 \\
\hline 0.924 & 0.930 & 0.924 & 0.918 & S3 \\
\hline \multirow[t]{4}{*}{0.0029} & & 0.0054 & & LSD 0.05 \\
\hline & 0.851 & 0.851 & 0.848 & MEAN F \\
\hline & & 0.0036 & & LSD 0.05 \\
\hline & & \multicolumn{3}{|c|}{ 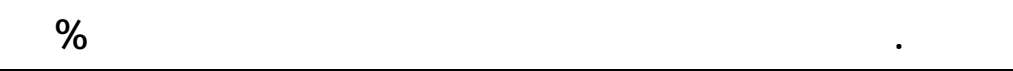 } \\
\hline MEAN .S & F3 $\left(40{\left.\mathrm{t} . h \mathrm{ha}^{-1}\right)}^{-1}\right.$ & F2 $\left(20 \mathrm{t} \mathrm{tha}^{-1}\right)$ & F1 $\left(0\right.$ t. ha $\left.^{-1}\right)$ & $\begin{array}{c}\text { Treatment }\left(\mathrm{mL} \cdot \mathrm{L}^{-}\right. \\
\left.1^{-}\right)\end{array}$ \\
\hline 0.289 & 0.293 & 0.289 & 0.285 & $\mathrm{~S} 1$ \\
\hline 0.305 & 0.309 & 0.304 & 0.302 & $\mathrm{~S} 2$ \\
\hline 0.332 & 0.336 & 0.332 & 0.329 & S3 \\
\hline \multirow[t]{4}{*}{0.0017} & & 0.0027 & & LSD 0.05 \\
\hline & 0.313 & 0.308 & 0.305 & MEAN. F \\
\hline & & 0.0017 & & LSD 0.05 \\
\hline & & \multicolumn{3}{|c|}{ جلط 9 ـ تأثير إضلفة للسماد العضوي في تركيز البوتلسيوفي النبلت\% } \\
\hline MEAN S & F3 (40 t .ha-1) & F2 (2o t .ha-1) & F1(0 t .ha-1) & treatment $\left(\mathrm{mL} \cdot \mathrm{L}^{-1}\right)$ \\
\hline 3.145 & 3.243 & 1.287 & 3.062 & $\mathrm{~S} 1$ \\
\hline 4.427 & 4.465 & 4.435 & 4.381 & S2 \\
\hline 5.102 & 5.174 & 5.109 & 5.022 & S3 \\
\hline
\end{tabular}




\begin{tabular}{|c|c|c|c|c|}
\hline 0.0071 & \multicolumn{3}{|c|}{0.0080} & LSD 0.05 \\
\hline & 4.294 & 4.224 & 4.155 & MEAN F \\
\hline & & 0.004 & & LSD 0.05 \\
\hline
\end{tabular}

\section{الاستنتالت}

من خلال ما قم عرضة من نتائج نستنتج بان لإض الفة الأسمدة العضويةسواء عن طريق الرش أوالإضلفة للتربة لإنة

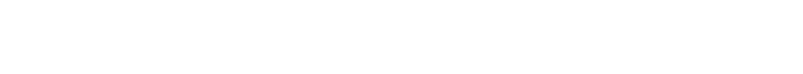
وتركيز العناصر الغذائية(النيتروجين والفنفور والبوتلسيوم) فئهية

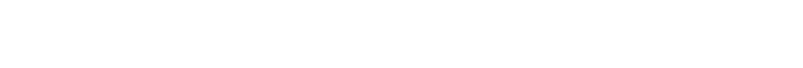

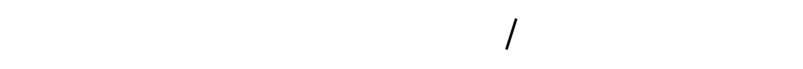

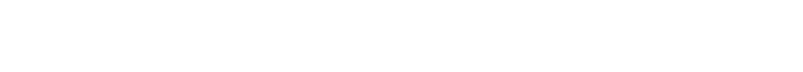

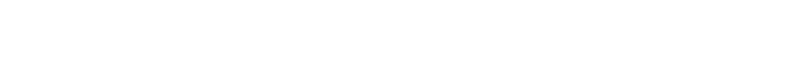
خواص التربة الفيزيائية والكيميائية، وان إضلفة اللماد رشا

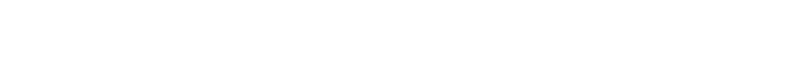

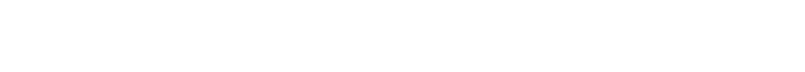

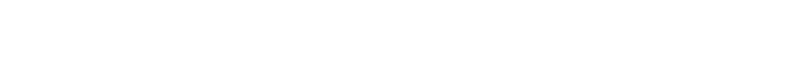

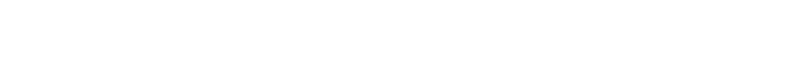

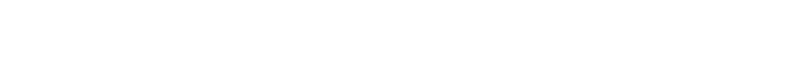

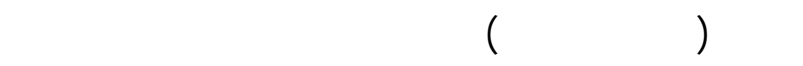

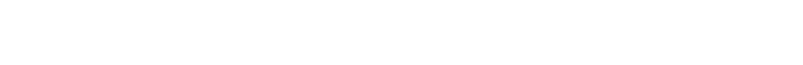

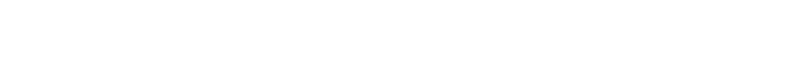

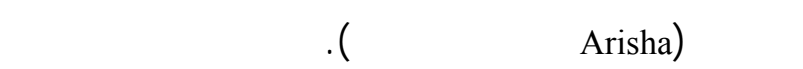

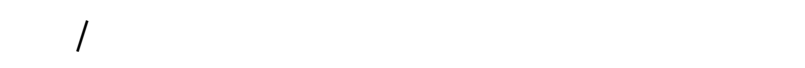

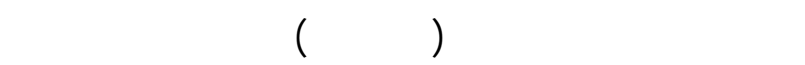

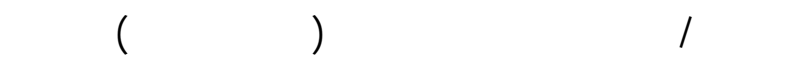

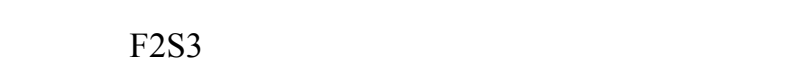

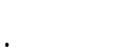

\section{الم ــالجع}

كما أن تدلخلات التسميد العضوي مطل الدرلمدة ق ق 2.

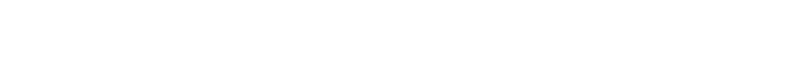

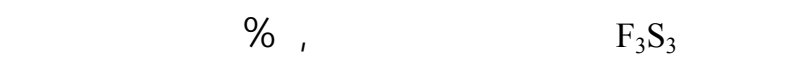
F2 F $_{2} \mathrm{~S}_{3}$

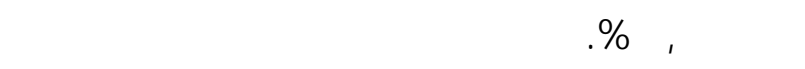

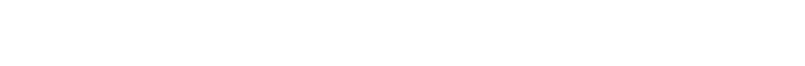

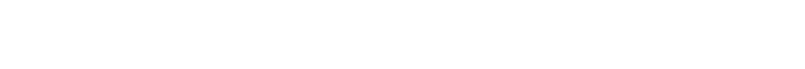

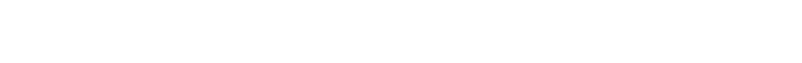

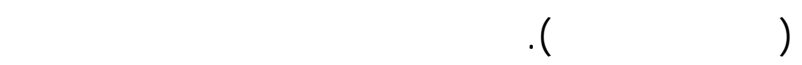

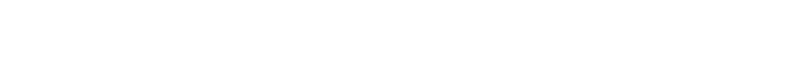

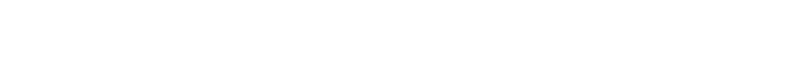
لمتصاص النبلت للعناص ـر الغذائي ـة وخاص ـة الف سنفور

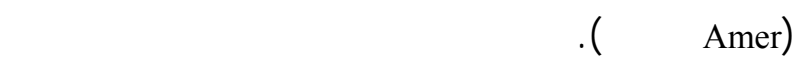

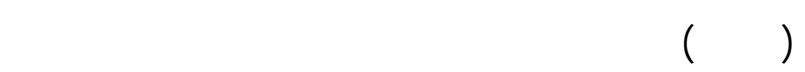

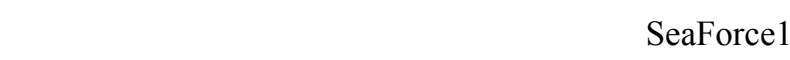

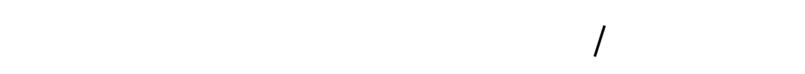

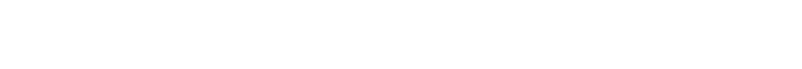
Abdel-Mawgoud بمستخلص الطحل بب البح ري (Ascophyllum nodosum)

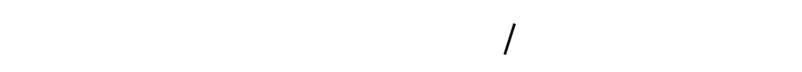

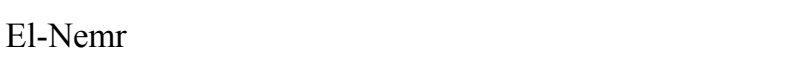

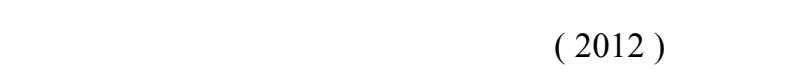

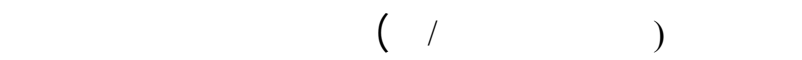
Beta-Alpha

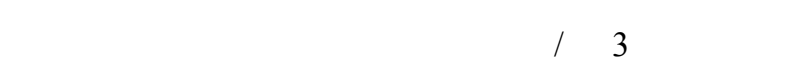

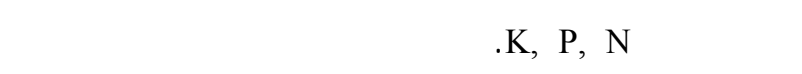

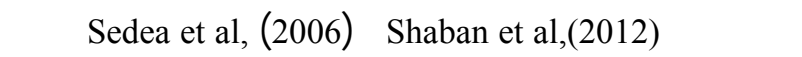
الطملطم والقاضي (بّا · r) على الذرة الشلمية. 
. مجلة الكوفة للعلوم الزراعي ـة مجل ــ 0 الع ددد (1) ص:I9V- IV9

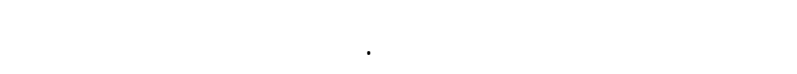

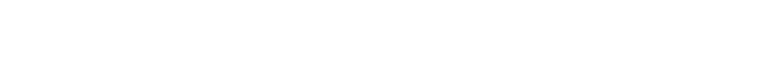

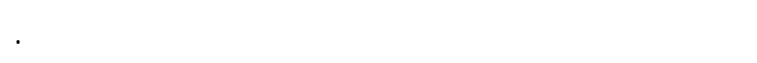
لُأروحة كتوره- كلية الزراعة - جلمعة بغداد.

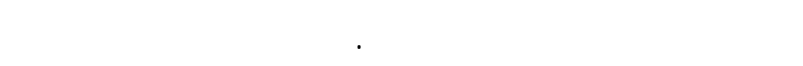

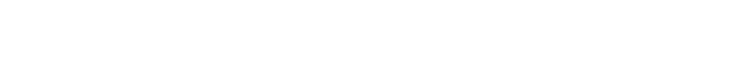

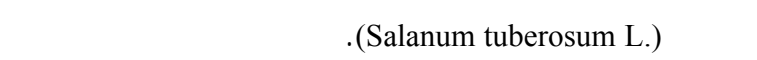

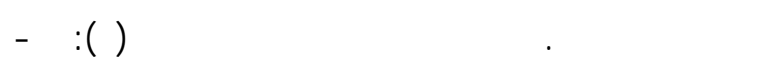

or

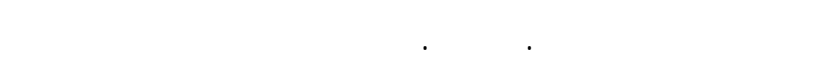

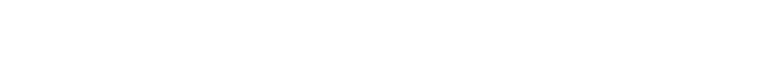
1. (Solanum tuberosum L.)

$$
.1 \varepsilon 0-1 \mu\rceil
$$

سعيد،ناصر عبادي. 7 - . r. تأثير التسميد الحيوي والنتروجيذ مي

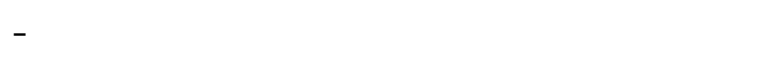
محلظة لحج. المجلة اليمنية للبحوث الزراعية- العدد الثالث

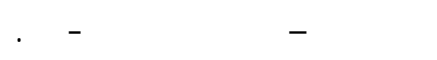

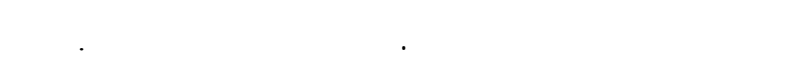

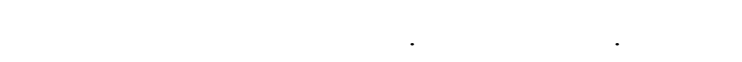

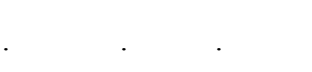

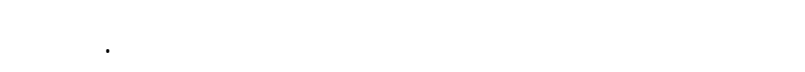

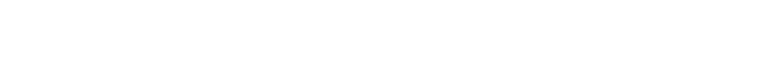
القرع (Cueurbtapepo L) . مجلة القاهسية للعلوم الزراعي ة.

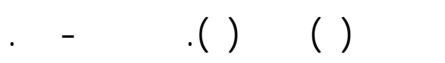

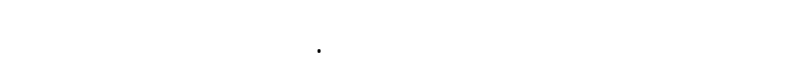

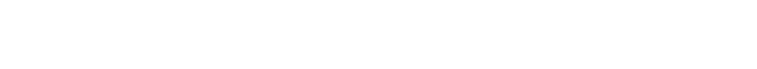

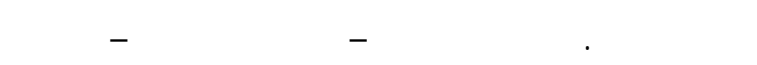
صنعاء.

الك ــلف.ح ـسين عب ــدالرحمن 997 ـ خ ــصوبة الترب ــة

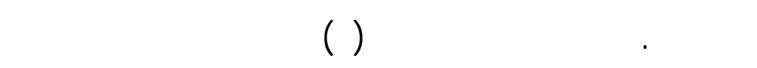
والشر. الجمهورية اليمنية.
أبو ضلحي، يوضف محمد ومؤيد لُممد اليونس 911 ـ دليل تغذنية

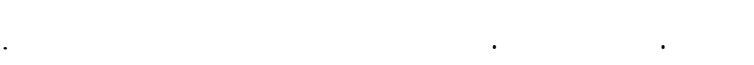
العراق.

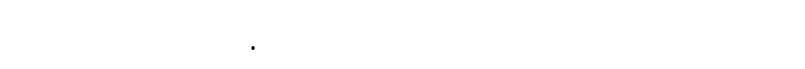
للمجموعة الثلمنة .الموارد الأرضية في الجمهورية اليمني ـة. المجلس الأعلى للحماية البيئية، صنعاء، اليمن.

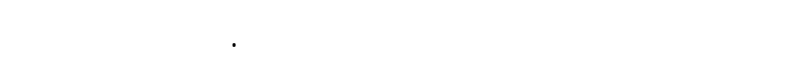
الهيومك والاعشب البحرية في نمو وازهار وحاطل الخيار - (Cucumis sativus L.)

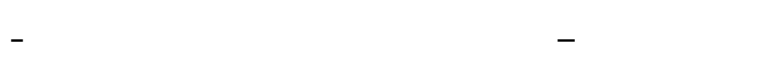
جمهورية العراق.

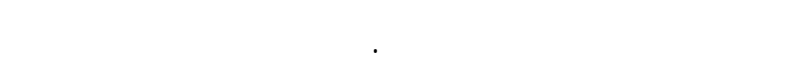

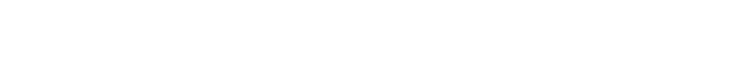

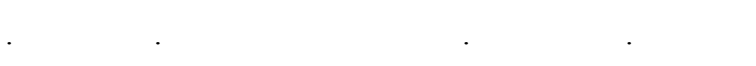
ص هسين، وفاء علي و بيلن حمزة مجيد 9 . . r. لستجابة صفين من

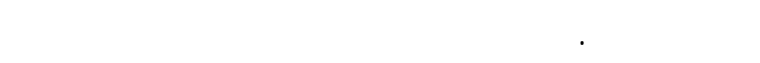

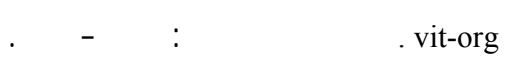

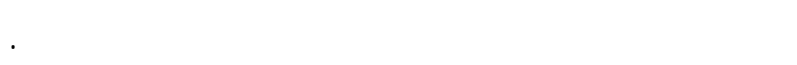

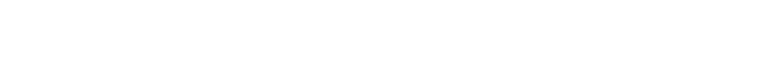

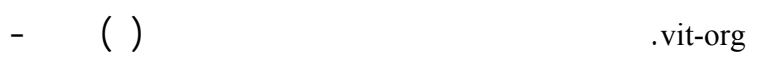
r.

حمزة، موله محمد ومسن علوانس لملن و عمرحم 2 عبي 2د

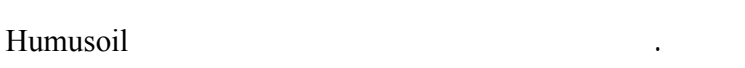

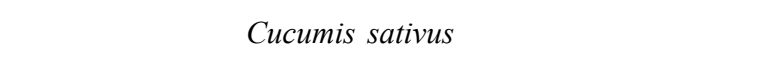
البيت الزجلجي.مجلة الفرات للعلوم الزراعية، ؟(1): عَ r

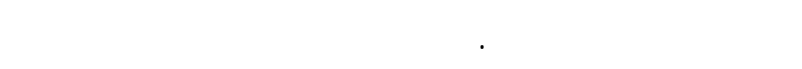

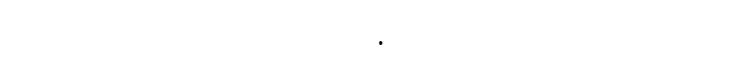

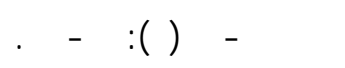
التحلف،سلمي علي عبدالمجيد، موسق محمدحمزة، حلم دعجل

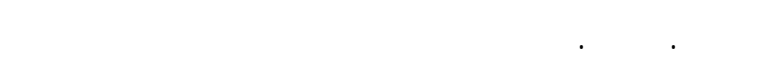

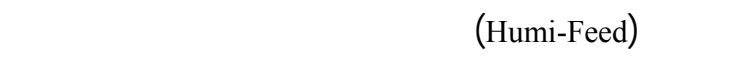


El-Nemr, M.A., M. El-Desuki, A.M. El-Bassiony and Z.F.Fawzy. 2012. Response of Growth andYield of Cucumber Plants (Cucumissativus L.) to Different FoliarApplications of Humic Acid and Biostimulators.Australian Journal of Basic and Applied Sciences, 6(3): 630-637.

ElSahookie, M. M. 2006. Geneticphysiologic and geneticmorphologic components in soybean . The Iraqi J.Agric. Sci. 37(2): 63-68.

Hamid , R. B.; S. Bidarigh; E. Azarpour, and R. Khosravi . 2012 . Effects of Natural Zeolite Application under Foliar Spraying with Humic Acid on Yield and Yield Components of Cucumber (Cucumis sativus L.). Inter. J.Agri Crop Sci., 4 (20) : 1485-1488.

El-Shabrawy, R. A.; A.Y. Ramadan and S. M. El-Kady . 2010 . Use of Humic Acid and some Biofertilizers to reduce Nitrogen rates on cucumber (Cucumis sativus L.) in relation to vegetative growth, yield and chemical composition. J. Plant Production, Mansoura University, Vol. 1(8): 1041 - 1051.

Jahan, Mohsen,AlirezaKoocheki, Mohammad-KazemTahami, Mohammad- ehzadAmiri and Mahdi NassiriMahallati.,2012, The Effects of Simultaneous Application of Different Organic and Biological Fertilizers on Quantitative and Qualitative Characteristics of Cucurbita pepo L. Journal of Life Sciences 6 .1145-1149 .

Karakurt Y.; H. Unlu; Halime Unlu and H. Padem . 2009. The influence of foliar and soil fertilization of humic acid on yield and quality of pepper. Acta Agriculturae Scandinavica, Plant Soil Science, 59 (3): 233 - 237.

Lester, G. E. and J. L. Jifon. 2007.Foliar applied potassium effects on cantaloupe quality . Acta Hort. 731:89-95.

Lopyes, R.; F. Cabera, E. madejan, F. Sancho and M. Alvares 2005 ). Urban compost as an Alternative for peat in Forestry Nursery growing Media Dynamic soil . Dynamic plant vol. 1Special Issue (9001) . Composts L pp 60-66.

Page,A.L.;R.h.Miller, and D.R.Keeney.1982.Method of Soil Analysis Part 2. chemical and microbiological properties. $2^{\text {nd }}$ edition. Amer. soc. of Agron. INC. Soil Sci.Soc.Amr.INC.Madison .Wisconsin.USA.

Sedea. Wafaa Mohamed Ahmed, J.H. Joseph and M.M.H. Kenawy 2006. Changes in soil physico-chemical properties and macronutrients contents of pea and tomato plants due to organic manure and some natural minerals fertilization. Mansoura University Journal. Agricultural Sciences. ISSN111: 7301-7316.

Shaban,Kh.A,Wafaa A. Hafez and Faten A. Kamar, 2012, Effect of Some Soil Amendments on Soil Fertility and Tomato Productivity under Saline Soil Condition. Minufiya J.Agric.Res.Vol.37 No. 4(2): 995-1005.
لكساد.المركز العربي لدرلسة المنطق الجلفة والأراضي القلحل ـة

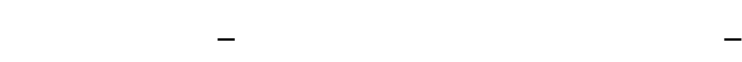

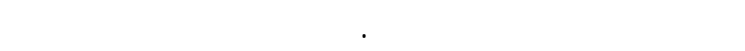
الأراضي في الجمهورية اليمنية.

كتلب الإحصاء الزراعي || ·r. الجمهورية اليمنية - وزارة

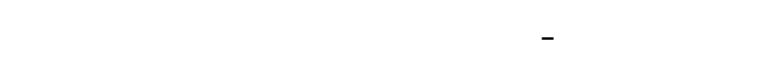
الزراعية.

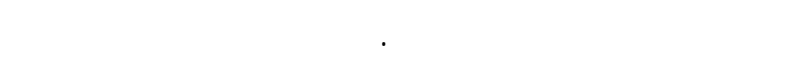

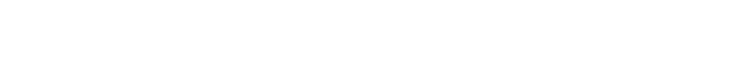

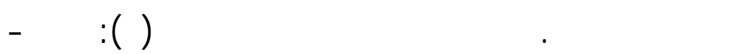

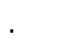

محمد،عبدارحيمسطلان وجليل لسكندر الصطيفو rا • r. ت اثير

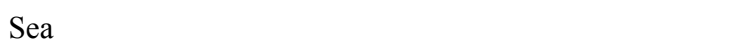
Force 1

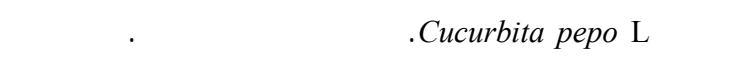

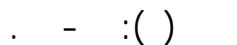

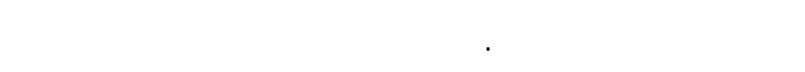
والحاصل وتشقق ثمارا لبطخ. مجلة العلوم الزراعية العراقية

$$
\text { .r.- 19:(દ)ع। - }
$$

Abdel.Mawgoud A. M.R; A. S. Tantaway Magdo M. Hafes and A. Hoda M. Habib (2010). Seaweed extract Improves growth, yield and quality of different watermelon hybrids. Research Journal of Agriculture and Biological Sciences . 6 ( 2 ) $161-168$.

Amer, A.S.S. (1981). Effect of Some Growth Regulators and Some Miner Elements on Growth and Yield of Tomako . M. Sc. Thesis. Faculity of Agric. Sci. / Moshhtohor / Zagazg Univ./ Egypt.

Arisha, H.M.E., A.A. Gad and S.E. Younes, 2003. Response of some pepper cultivars to organic and mineral nitrogen fertilizer under sandy soil conditions. Zagazig J. Agric. Res., 30:1875- 1899.

Dilson, A.B. (2002). Origin and evolution of cultivated cucurbita. Ciencia Rural, 32 (5): 715-723. 


\title{
SUMMARY \\ Effect of Organic Fertilizer Application to the Soil or Spraying Plant in Qualitative Characteristics and Mineral Content of Squash
}

\author{
Najeeb Mohamed Hussein Al-Magrebi
}

To study the effect of adding organic fertilizer to the soil or spraying plant in qualitative characteristics and mineral content of the plant Squash. An experiment was carried out in the greenhouse of the department of Soil and water Faculty of Agriculture - Sana'a University in the spring season of 2010 in the soil of sedimentary Silty Clay loam used plastic pots for planting experiment added soil to pots $10 \mathrm{~kg}$ soil / pot. The experiment was designed using a split plot design with three replications. The ground organic fertilizing called the Pew Ho Moses component (25\% humic substances and NPK and trace elements $(\mathrm{Mn}, \mathrm{Ca}, \mathrm{B}, \mathrm{Mo}, \mathrm{Zn}, \mathrm{Co}$, $\mathrm{Fe}$ ) main pieces and add to the soil in three levels, namely, $(0,20.40) \mathrm{t} / \mathrm{ha}$ and have been given the following codes (F1, F2, F3), respectively, add compost ground into the soil and mix well before planting by the addition levels. organic fertilizer spray, called Homistar component of the same former fertilizer components and concentrations less sub-blocks in three levels, namely, $(0,20,40) \mathrm{me} / \mathrm{L}$, was added to plants by spraying and have been given the following codes (S1, S2, S3) to levels previously mentioned was spraying fertilizer organic liquid on plants in two installments the first spray after 21 days of germination and the second after 15 days Spatter from the first by the addition of various levels, were planted the seeds of the plant Squash (Alzukina) class, was added stimulus of nitrogen fertilizer on the form of urea $46 \% \mathrm{~N}$ at a rate of $30 \mathrm{~kg} \mathrm{~N} /$ ha for all treatments. Spraying the plant by organic fertilizers increased fresh weightm and dry matterm significantly. Mean while, increased TSS andNPK content especially at the third level $40 \mathrm{~mL} . \mathrm{L}^{-1}$.

Keywords: organic fertilizer, Squash, soluble solids, dry matter 